\title{
Phytoprotection
}

\section{Prix et hommages du centenaire de la Société de protection des plantes du Québec}

\section{Danielle Bernier, Sylvie Rioux et Daniel Dostaler}

Volume 89, numéro 2-3, décembre 2008

Journée commémorative du centenaire de la société de protection des plantes du Québec

Centennial Commemoration of the Québec Society for the Protection

of Plants

URI : https://id.erudit.org/iderudit/038247ar

DOI : https://doi.org/10.7202/038247ar

Aller au sommaire du numéro

Éditeur(s)

Société de protection des plantes du Québec (SPPQ)

ISSN

0031-9511 (imprimé)

1710-1603 (numérique)

Découvrir la revue

Citer ce document

Bernier, D., Rioux, S. \& Dostaler, D. (2008). Prix et hommages du centenaire de la Société de protection des plantes du Québec. Phytoprotection, 89(2-3),

143-164. https://doi.org/10.7202/038247ar d'utilisation que vous pouvez consulter en ligne. 


\title{
Prix et hommages du centenaire de la Société de protection des plantes du Québec
}

\author{
Danielle Bernier ${ }^{1,2}$, Sylvie Rioux ${ }^{2,3}$ et Daniel Dostaler ${ }^{2,4,5}$
}

PHYTOPROTECTION $89: 143-164$

La Société de protection des plantes du Québec (SPPQ) a été fondée le 24 juin 1908, au Collège Macdonald de I'Université McGill, par le professeur William Lochhead, "a long-time president of the Québec Society for the Protection of Plants, the oldest such society in the world to be continuously functioning under its original name" (Estey 1983). Au fil de ces 100 années (1908-2008), des personnes remarquables ont ponctué l'histoire de la SPPO et l'histoire de la protection des cultures et des forêts du Québec et du Canada. Moment fort de la journée commémorative du centenaire de la Société: les prix et hommages décernés à des pionniers de la SPPQ, à des services publics et à des leaders qui ont contribué à l'avancement de la protection des plantes et au rayonnement de la Société.

Les trois lauréats des prix Excellence pour l'ensemble des réalisations - Lifetime Achievement Award, Ralph H. Estey, Gilles Émond et Guillemond B. Ouellette, représentent, d'une part, la force pionnière, I'innovation, le leadership et, d'autre part, la mémoire de notre Société. Ces lauréats ont marqué la nématologie, la malherbologie et la phytopathologie, autant au plan de l'administration et de l'enseignement que de la recherche universitaire ou publique. De même, les noms des trois lauréats des prix Hommage, Léon-M. Tartier, Claude Richard et Gilbert Banville, évoquent la permanence et le service au sein de notre Société. Les trois récipiendaires sont des pionniers et des leaders de la phytopathologie.

Les quatre premiers lauréats d'un hommage, Anne Légère, Conrad Cloutier, Carole Beaulieu et Guy Boivin, illustrent bien ce que devrait être notre Société, soit une association des chercheurs scientifiques, hommes et femmes, qui sont des forces vives dans tous les champs de recherche et d'application de la phytoprotection (malherbologie, entomologie et phytopathologie) en centre de recherche ou à l'université. Des hommages ont aussi été attribués à quatre chercheurs ayant œuvré en pathologie forestière et agricole: Louis Bernier, Richard Hamelin, André Lévesque et Richard Bélanger. Ce sont des pionniers et forces vives du développement d'approches analytiques de laboratoire et d'outils moléculaires. On leur doit des avancées fondamentales et appliquées en résistance génétique, en écologie moléculaire et en lutte biologique en phytopathologie. Ces chercheurs contribuent de façon remarquable au rayonnement international de notre Société.

L'hommage Pierre-O.-Thibodeau a été remis à deux membres du Comité permanent de nomenclature française des maladies des plantes, Luc Couture et Jean Denis Brisson, pour souligner l'excellence du travail de l'ensemble des membres de ce comité dédié à la publication Noms des maladies des plantes au Canada. L'hommage J.-Champlain-Perrault/ElzéarCampagna a pour sa part été décerné à trois services publics en phytoprotection, soit le Service des relevés et des diagnostics (MNRF), le Laboratoire de diagnostic en phytoprotection du Québec (MAPAQ) et le Réseau d'avertissements phytosanitaires (MAPAQ), pour souligner la contribution remarquable et essentielle du personnel de ces services publics à la protection des cultures et des forêts du Québec.

Enfin, I'hommage Victor-A.-Huard/Georges-Maheux a été décerné à Jacques Régnière, entomologiste forestier, lors du Symposium "La phytoprotection, 100 ans de découvertes: un tremplin vers I'avenir!", le 21 novembre 2008, tenu dans le cadre de la Réunion annuelle conjointe SPPQ-SEQ. Cet hommage en entomologie forestière scelle les liens qui unissent la Société de protection des plantes du Québec et la Société d'entomologie du Québec en cette année du centenaire de la SPPO.

Le comité du centenaire et le comité des prix et hommages ont suivi un fil conducteur dans la dénomination des prix et hommages, dans le choix des lauréats et celui des personnes invitées à présenter les prix et hommages aux lauréats: pionnier et leader; rôle et engagement au sein de la Société; rayonnement de la Société et de la phytoprotection; caractère multidisciplinaire de la Société, des champs et des disciplines de la phytoprotection, tant en agriculture qu'en foresterie; souci de représentativité des services publics et des institutions d'enseignement et de recherche québécois en protection des plantes.

Our Society has changed. However, its early beginnings remain some of its most notable moments. For all his historical research, which was essential to the preparation of the awards and honours of this Centennial Celebration, we express to Dr. Ralph $\mathrm{H}$. Estey our most profound gratitude.

1. Direction de l'innovation scientifique et technologique, ministère de l'Agriculture, des Pêcheries et de l'Alimentation, Québec (Québec).

2. Membre du comité des prix et hommages, centenaire de la Société de protection des plantes du Québec.

3. Centre de recherche sur les grains inc. (CÉROM), Québec (Québec).

4. Département de phytologie, Université Laval.

5. Les auteurs remercient chaleureusement les lauréats et les personnes invitées à présenter les prix et hommages pour leur contribution inestimable à la préparation de ce texte. 


\section{Excellence pour l'ensemble des réalisations/Lifetime Achievement \\ Prix de la Société de protection des plantes du Québec/ Québec Society for the Protection of Plants Award

\author{
Ralph Howard Estey
}

\begin{abstract}
"Ralph Howard Estey has deserved and is assured of a place in the history of plant pathology in Québec and in Canada. He richly deserves the honour the Québec Society for the Protection of Plants does to him, and to itself, by naming him an Honorary Member."
\end{abstract}

W.E. Sackston (1986)

“In 1960, Dr. Estey offered a degree-credit course in nematology, the first at any Canadian university. This pioneer work was recognized by the North Atlantic Treaty Organization, which awarded him a scholarship to attend, and to participate in, its international school of nematology in Bari, Italy. [...] It should also be mentioned that Dr. Estey is one of the founding members of the American Society of Nematology. He is a lifetime member of the European Society of Nematologists."

The Canadian Phytopathological Society (2003)

Professeur émérite du Plant Science Department du Collège Macdonald de I'Université McGill, Ralph Howard Estey, président de la Société de protection des plantes du Québec (SPPQ) en 1963-1964, est membre honoraire de la Société depuis 1986. II fut aussi rédacteur associé de la revue Phytoprotection durant de nombreuses années. Le Dr Estey est, entre autres, "Fellow" de la Linnean Society of London depuis 1971 et "Fellow" de la Société canadienne de phytopathologie (SCP) depuis 1986, dont il fut président en 1978-1979. C'est sous sa présidence que le Canadian Journal of Plant Pathology a été créé. La SCP a octroyé au Dr Estey le prix Excellence pour I'ensemble des réalisations - Lifetime Achievement Award en 2003. Le Dr Estey est un pionnier hors du commun de l'enseignement et de la recherche en mycologie et en phytopathologie, en particulier dans le domaine de la nématologie. II a enseigné la phytopathologie, la nématologie et la botanique, en plus de créer un cours intitulé "Histoire de la phytopathologie". Le Dr Estey occupe une place d'honneur dans I'histoire de la protection des plantes au Canada. Les citations de Sackston' et de la Canadian Phytopathological Society ${ }^{2}$ sont éloquentes à cet égard.

Le Dr Estey est reconnu pour la qualité inestimable de ses écrits sur l'histoire de l'agriculture, de la protection des cultures, de la mycologie et de la phytopathologie, dont: "History and Perspective of Phytopathology in Québec" (1970), "Histoire de la Société de protection de plantes du Québec" (1983), Essays on the Early History of Plant Pathology and Mycology in Canada (1994), "History of Mycology in Canada " (1994), " 150 years of teaching plant pathology at McGill University" (2007) et la conférence " 100 years with the Québec Society for the Protection

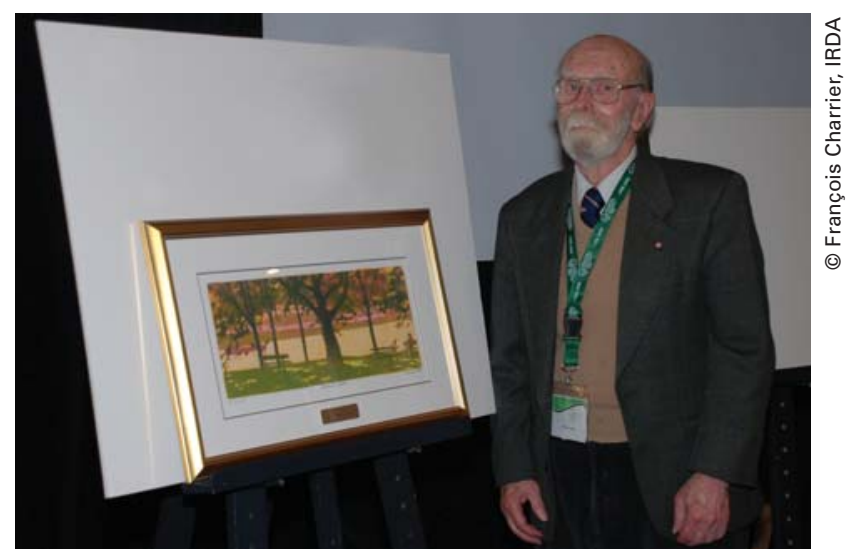

Ralph Howard Estey

Emeritus Professor in the Department of Plant Science of Macdonald College at McGill University, Ralph Howard Estey, president of the Québec Society for the Protection of Plants (QSPP) in 1963-1964, is an honorary member of the QSPP since 1986. Dr. Estey was also an associate editor of Phytoprotection for several years. Dr. Estey is Fellow of the Linnean Society of London since 1971 and Fellow of the Canadian Phytopathological Society (CPS) since 1986. It was under his term as president of the CPS in 1978-1979 that the Canadian Journal of Plant Pathology was launched. The CPS honoured Dr. Estey with a Lifetime Achievement Award in 2003. Dr. Estey is a pioneer teacher and researcher in mycology, phytopathology and nematology. He taught plant pathology, nematology and botany, and he created a course entitled "History of Plant Pathology". Dr. Estey is assured of a place in the history of plant protection in Canada. The citations of Sackston $^{1}$ and of the Canadian Phytopathological Society $^{2}$ speak for themselves.

Dr. Estey is recognized for his remarkable notices, papers and books on the history of agriculture, plant protection, mycology and phytopathology, including: "History and Perspective of Phytopathology in Québec" (1970), Essays on the Early History of Plant Pathology and Mycology in Canada (1994), "History of Mycology in Canada" (1994), "150 years of teaching plant pathology at McGill University" (2007), and the conference "100 years with the Québec Society for the Protection of Plants" (Quebec City, November 19, 2008). Dr. Estey has published notices and commentaries on Canadian plant pathologists and entomologists in journals such as Phytoprotection, the Canadian Journal of Plant Pathology and the Annual Review of Phytopathology. His historical works were 
of Plants" (Québec, le 19 novembre 2008). Le Dr Estey a publié des articles sur des phytopathologistes et des entomologistes canadiens, entre autres dans les revues Phytoprotection, Canadian Journal of Plant Pathology et Annual Review of Phytopathology. Ses écrits ont permis de préparer et de dédier les hommages de la journée commémorative du centenaire de la Société aux pionnières Margaret Newton (première femme membre de la Société; Bourse du centenaire SPPQ 2008 Margaret-Newton) et Carrie Derick, ainsi qu'aux pionniers Elzéar Campagna, James Fletcher, William-Pollock Fraser, Henri Généreux, Victor-A. Huard, René-O. Lachance, William Lochhead, Georges Maheux, J.-Champlain Perrault, René Pomerleau, Jacques Simard et Douglas Weir.

À l'occasion de la commémoration de son centenaire (1908-2008), la Société de protection des plantes du Québec est heureuse de présenter au Dr Ralph Howard Estey le prix Excellence pour I'ensemble des réalisations - Lifetime Achievement Award. La Société de protection des plantes du Québec souligne ainsi la contribution inestimable du Dr Estey au rayonnement de la Société et de la revue Phytoprotection et lui exprime ses plus sincères remerciements.

Prix Excellence pour l'ensemble des réalisations Lifetime Achievement Award remis par monsieur Guy Bélair, vice-président de la Société en 2007-2008 et président en 2008-2009; chercheur au Centre de recherche et de développement en horticulture d'Agriculture et Agroalimentaire Canada (AAC) à Saint-Jean-sur-Richelieu; pionnier et leader de la recherche en nématologie, notamment sur les moyens de lutte non chimiques et la lutte biologique, sur la biologie du nématode doré Globodera rostochiensis, sur l'effet du millet à chandelle sur le Pratylenchus penetrans chez la pomme de terre, sur les nématodes entomopathogènes et sur la lutte raisonnée aux maladies des plantes horticoles. central in the preparation of the awards and honours of the Centennial Celebration of the QSPP. The awards and honours are dedicated to the pioneers Margaret Newton (first woman member of the Society; 2008 QSPP Margaret-Newton Centennial Fellowship), Carrie Derick, Elzéar Campagna, James Fletcher, William-Pollock Fraser, Henri Généreux, Victor-A. Huard, René-O. Lachance, William Lochhead, Georges Maheux, J.-Champlain Perrault, René Pomerleau, Jacques Simard and Douglas Weir.

On the occasion of its Centennial Celebration (19082008), the Québec Society for the Protection of Plants is pleased to honour Dr. Ralph Howard Estey. His outstanding achievements and contributions to plant pathology and nematology, Phytoprotection and the Society are recognized through this Lifetime Achievement Award. A gift is also presented to Dr. Estey to express the Society's profound gratitude

1 Sackston, W.E. 1986. Dr. R.H. Estey, named Honorary member of the Québec Society for the Protection of Plants. Phytoprotection 67: 79-80.

2 Canadian Phytopathological Society. 2003. Lifetime Achievement - The Canadian Phytopathological Society Award - Ralph Howard Estey. Can. J. Plant Pathol. 25: 454-457. 


\section{Excellence pour l'ensemble des réalisations}

\section{Prix de la Société de protection des plantes du Québec \\ Gilles Émond}

"Son passage a profondément marqué le paysage de la protection des plantes au Québec. Son entrain et son dynamisme sont des traits de sa personnalité qui lui ont permis de s'attacher l'amitié autant que la fidélité de ses collaborateurs. [...] Gilles Émond fait partie de ces gestionnaires visionnaires qui savent créer des instruments majeurs. Ses efforts ont concouru à installer un support scientifique pour permettre aux producteurs du Québec de lutter efficacement contre les ennemis des cultures."

La Société canadienne de phytopathologie (1996)

Gilles Émond a complété un baccalauréat en sciences agronomiques à l'Université de Montréal il y a 50 ans. Un peu plus tard, il a poursuivi ses études à I'Université McGill où il a obtenu le premier doctorat en malherbologie au Québec. Il a enseigné le premier cours en malherbologie à la Faculté des sciences de I'agriculture et de l'alimentation (FSAA) de I'Université Laval. Le Dr Émond a travaillé au ministère de I'Agriculture, des Pêcheries et de l'Alimentation du Québec (MAPAQ) pendant 37 ans. Il a dirigé, pendant plus de 25 ans, le Service de recherche en protection des cultures à la Direction de la recherche de ce ministère. Dans le cadre de ses fonctions comme gestionnaire, il a été le maître d'œuvre de plusieurs réalisations majeures, dont la mise sur pied du Réseau d'avertissements phytosanitaires du Québec, du Laboratoire de diagnostic en phytoprotection du Québec et d'un complexe phytotronique informatisé. II a été président de plusieurs comités liés à la malherbologie, aux pesticides et à la protection des plantes. II est l'auteur de plusieurs publications et a participé à plusieurs missions en France. Son dévouement, son leadership et ses nombreuses réalisations en protection des plantes ont été soulignés par la Société et également par la Société canadienne de phytopathologie en le nommant membre honoraire. II a aussi reçu le titre de Commandeur de l'Ordre du Mérite agronomique décerné par l'Ordre des agronomes du Québec.

Sa contribution au rayonnement de la SPPQ et son rôle au sein de la Société ont été très importants. II en a assuré la présidence en 1972-1973. Peu après sa retraite, le Dr Émond a pris les rênes de la revue Phytoprotection comme rédacteur en chef, et ce, pour 10 ans (1995-2005). C'est sous sa direction que la revue prend son format et son apparence actuels, que de nouvelles rubriques apparaissent et que les versions numériques voient le jour. Le Dr Émond a aussi présidé le Comité permanent de nomenclature française des maladies des plantes au Canada pendant 15 ans. Le travail très exigeant de ce comité s'est concrétisé par la parution de deux nouvelles éditions du volume Noms des maladies des plantes au Canada, en plus d'une édition corrigée. Sa contribution ne s'arrête pas là : il a créé et présidé le comité du centenaire de la Société qui s'est chargé de souligner

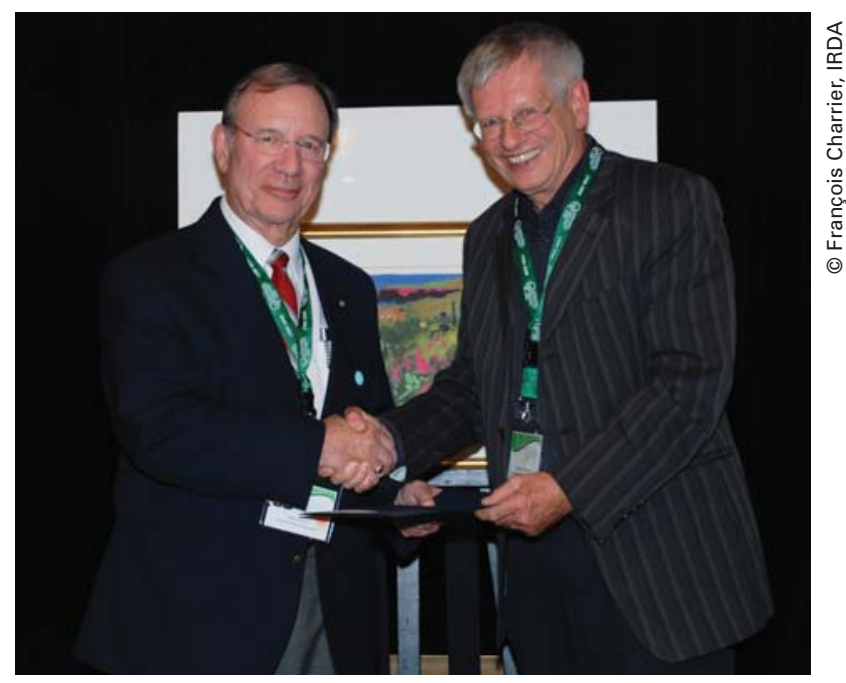

Gilles Émond et Peterjürgen Neumann

cet anniversaire, dont l'organisation de la journée commémorative.

À l'occasion de la commémoration de son centenaire (1908-2008), la Société de protection des plantes du Québec est fière de présenter au Dr Gilles Émond le prix Excellence pour l'ensemble des réalisations. La Société souligne ainsi la contribution inestimable du Dr Émond à son rayonnement ainsi qu'à celui de la revue Phytoprotection et lui exprime ses plus sincères remerciements.

Prix Excellence pour l'ensemble des réalisations remis par le Dr Peterjürgen Neumann, président (1990-1991) et membre honoraire de la Société (2005); professeur honoraire de l'Université de Montréal; pionnier et leader de la recherche en mycologie et en phytopathologie, notamment en biologie et en taxonomie (nombreuses premières mentions mycologiques) de champignons phytopathogènes et de champignons mycorhiziens. 


\section{Excellence pour l'ensemble des réalisations}

\section{Prix de la Société de protection des plantes du Québec \\ Guillemond B. Ouellette}

\begin{abstract}
"Guillemond B. Ouellette is nationally and internationally known for his outstanding research in forest pathology. $\mathrm{He}$ is a leader and an innovative researcher in cytochemistry and fine structure of fungal pathogens, in morphology and taxonomy of wood inhabiting fungi, and in the use of advanced technologies in applied pathology."
\end{abstract}

The Canadian Phytopathological Society (1997)

"Guillemond Ouellette ignore l'expression “Ce n'est pas possible à faire". C'est certainement un des chercheurs les plus innovateurs et un esprit créatif d'idées nouvelles en recherche. II ne craint pas de remettre en question des concepts bien établis, dans le seul but de repousser les limites de la connaissance."

Pierre Mathieu Charest

Guillemond Ouellette a complété un baccalauréat en sciences à I'Université de Moncton en 1952. II a poursuivi ses études à I'Université Laval (La Pocatière) et à Cornell University où il a obtenu un doctorat en 1960 sous la supervision du Dr Donald S. Welch. Guillemond Ouellette a œuvré durant toute sa carrière au Centre de foresterie des Laurentides (CFL) du Service canadien des forêts (SCF), à Québec. À deux reprises, soit en 1968-1969, au laboratoire du professeur Emil Müller, et en 1990, le Dr Ouellette a effectué des séjours de recherche au Swiss Polytechnical Institute (ETH) de Zürich (École Polytechnique Fédérale) à titre de professeur invité. Leader de la taxonomie et de la morphologie des champignons, le Dr Ouellette compte plusieurs réalisations notoires: la découverte des "microhyphae" fongiques dans les tissus infectés (Ceratocystis ulmi, syn. Ophiostoma novo-ulmi); la découverte de quelques maladies des arbres, surtout en plantation, et des précisions sur des cycles infectieux (ex. Hypoxylon sp.); et la description de nouveaux genre (Lophomerum) et espèces (ex. Phacotheca dimorphospora). Le Dr Ouellette est un leader par ses travaux sur le chancre scléroderrien (Gremmeniella abietina (Lagerberg) Morelet var. balsamea Petrini, L.E. Petrini, Laflamme, Ouellette, et var. abietina Petrini, L.E. Petrini, Laflamme, Ouellette). II est reconnu comme pionner et force vive des techniques de marquage en histopathologie et aspects ultrastructuraux et de I'utilisation d'anticorps mono- et polyclonaux en pathologie forestière, ainsi que de la recherche sur les mécanismes de résistance chez des plantes hôtes ou non-hôtes. Membre du Centre de recherche en biologie forestière, il a été rédacteur associé des revues Phytopathology et Canadian Journal of Botany. II a aussi agi à titre de consultant concernant des problèmes affectant les arbres et arbustes ornementaux dans la ville de Québec, entre autres. Professeur associé à l'Université Laval, il y a enseigné la mycologie et la physiologie des champignons et

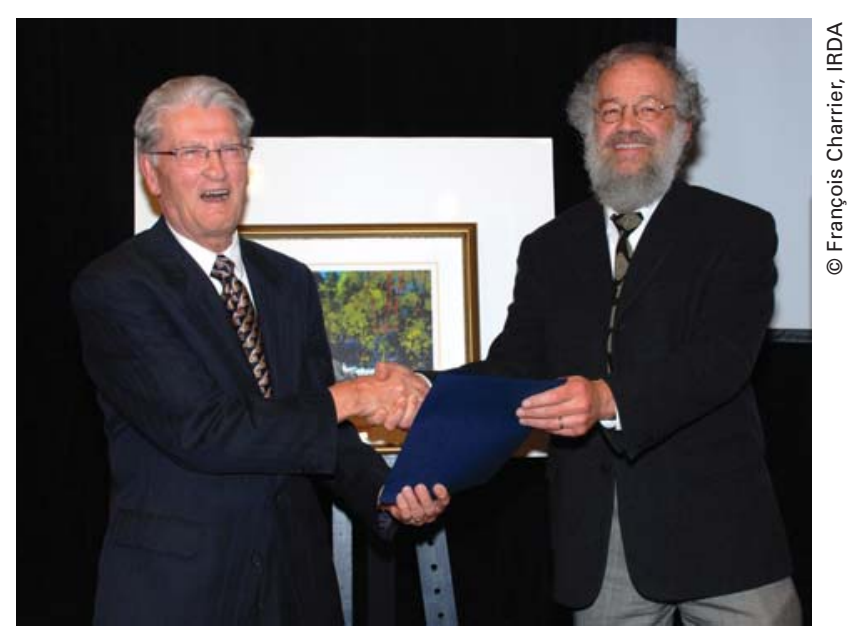

Guillemond Ouellette et Gaston Laflamme

supervisé des étudiants aux cycles supérieurs. Avec l'aide d'étudiants et d'autres chercheurs, des recherches ont aussi été menées sur la tomate, I'aubergine et l'œillet. Guillemond Ouellette a toujours été vivement apprécié par ses collègues et ses étudiants pour sa grande gentillesse et son humanité, sa disponibilité sans réserve et sa capacité de travail hors du commun.

Pour sa contribution au rayonnement de la Société et pour son rôle au sein de la SPPQ dont il fut le président en 1979-1980, le Dr Ouellette a été reçu membre honoraire en 1993. II est aussi récipiendaire du prix de l'Excellence scientifique de la Société canadienne de phytopathologie (1995), du Canadian Research Award et du prix René-Pomerleau pour ses travaux en arboriculture. Chercheur émérite, il est l'auteur de très nombreuses publications. II est co-rédacteur des livres Host Wall Alterations by Parasitic Fungi (VI Congrès international de phytopathologie, Montréal, 1993) et New Approaches to Elm Conservation ( $2^{\circ}$ Conférence internationale sur la maladie hollandaise de I'orme). Après sa retraite en 1995, Guillemond Ouellette a poursuivi ses recherches au CFL durant 11 autres années. Fait remarquable, de 1995 à 2007, le Dr Ouellette "retraité " a publié plus de 20 articles, dont huit dans la revue Phytoprotection. II a récemment publié un texte, avec la collaboration de Pierre Mathieu Charest et d'Hélène Chamberland, dans la rubrique "Forum " de Phytoprotection (2007, vol. 88: 77-81). On y expose de façon succincte le fil conducteur de la réalisation du livre, du DVD et du site Web (http://www.wilt-ism.net/page1.aspx) intitulés Closeup on some aspects of fungal wilt diseases portant sur les maladies de flétrissement, avec une attention particulière à la maladie hollandaise de l'orme.

À l'occasion de la commémoration de son centenaire (1908-2008), la Société de protection des plantes du Québec est fière de présenter au Dr Guillemond 
B. Ouellette le prix Excellence pour l'ensemble des réalisations. La Société de protection des plantes du Québec souligne ainsi la contribution remarquable du Dr Ouellette à son rayonnement et lui exprime ses remerciements les plus sincères.

Prix Excellence pour l'ensemble des réalisations remis par le Dr Gaston Laflamme, président de la Société en 2001-2002; chercheur au Centre de foresterie des Laurentides (CFL) du Service canadien des forêts (SCF) à Québec; pionnier et leader de la recherche en pathologie forestière, notamment sur les maladies des arbres en plantation, sur la taxonomie de champignons ascomycètes pathogènes des arbres, l'épiphytie et les relations hôte-parasite de maladies des pins et des épinettes, ainsi que sur la lutte sylvicole et biologique contre les maladies des pins rouges et des pins blancs.

\title{
Prix Hommage Douglas-Weir/Marc-André Richard
}

\section{Secrétariat et service au sein de la Société de protection des plantes du Québec}

\author{
Léon-M. Tartier
}

Léon Tartier a obtenu un baccalauréat en sciences agronomiques de I'Université de Montréal et une maîtrise en phytopathologie de I'Université McGill. Léon Tartier a passé toute sa carrière, soit 34 années, au Service de phytotechnie de Saint-Hyacinthe du ministère de I'Agriculture, des Pêcheries et de I'Alimentation du Québec (MAPAQ). Ses travaux de recherche ont porté en grande partie sur les maladies de la pomme de terre et, particulièrement, le mildiou (Phytophthora infestans) et la verticilliose (Verticillium albo-atrum et $V$. dahliae). II a entre autres travaillé de concert avec le Réseau d'avertissements phytosanitaires au développement et au raffinement d'un système prévisionnel du mildiou. II a aussi recherché des moyens de lutte aux maladies des légumes en sols organiques, tel le charbon de I'oignon (Urocystis magica), et à la tavelure du pommier (Venturia inaequalis).

II y a 10 ans, Léon Tartier prenait sa retraite. II a depuis fondé la compagnie PHYTOPROTECTION 2000 au sein de laquelle il travaille comme consultant. II y procède, pour différentes compagnies, à des tests d'efficacité de nouveaux fongicides pour lutter contre le mildiou et d'autres maladies de la pomme de terre en vue de I'homologation de ces produits. II évalue aussi la sensibilité au mildiou de nouvelles lignées de pomme de terre.

Léon Tartier est membre honoraire de la Société depuis 1998. II a été rédacteur des premiers bulletins de nouvelles de la Société (1977-1980), prédécesseurs des Échos Phytosanitaires. II a participé aux travaux du Comité permanent de nomenclature française des maladies des plantes qui ont mené aux $2^{\mathrm{e}}(1975), 3^{\mathrm{e}}$ (1991) et $4^{\mathrm{e}}$ (2003) éditions de l'ouvrage Noms des maladies des plantes au Canada. II a aussi été, et c'est là une contribution hors du commun, secrétaire de la Société pendant plus de 30 ans, soit pendant près du tiers de l'existence de la Société. À ce titre, il a par-

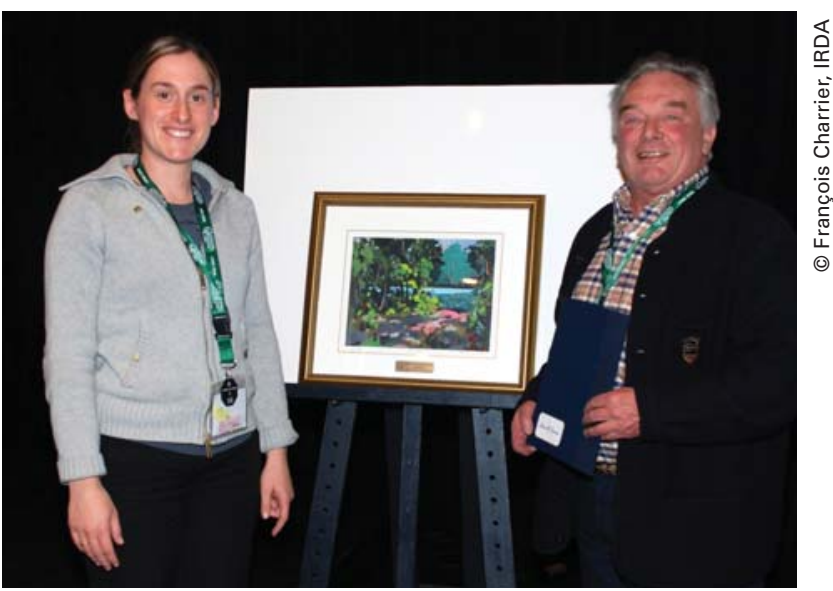

Geneviève Richard et Léon-M. Tartier

ticipé à toutes les activités de la Société. Il a d'ailleurs été un collaborateur essentiel du comité organisateur de la journée commémorative du centenaire.

En cette Année internationale de la pomme de terre (http://www.potato2008.org/fr/index.html) et à l'occasion de la journée commémorative de son centenaire (1908-2008), la Société de protection des plantes du Québec est fière de présenter le prix Hommage Douglas-Weir ${ }^{1} /$ Marc-André Richard ${ }^{2}$ à $M$. Léon-M. Tartier en gage de reconnaissance pour ses nombreuses années de service et de dévouement et sa contribution inestimable à l'administration de la Société.

Prix Hommage remis par M. Marc-André Richard et par Mme Geneviève Richard, actuelle secrétaire de la Société de protection des plantes du Québec et professionnelle de recherche à I'Institut de recherche et de développement en agroenvironnement (IRDA) à Saint-Hyacinthe.

1. Douglas Weir. Membre fondateur de la Société et premier secrétaire-trésorier de la SPPO de 1908 à 1911 . Entomologiste, étudiant du président-fondateur de la SPPQ, William Lochhead.

2. Marc-André Richard. Agronome. Président de la Société en 1981-1982. Assistant secrétaire de la Société de 1961 à 1967. II a réalisé la compilation de la liste des 684 articles publiés dans tous les Rapports annuels de la SPPO de 1908 à 1961 . Richard, M.A. 1966. Rapports annuels de la Société de Québec pour la protection des plantes (1908-1961). Phytoprotection 47 (1): 1-29. 


\section{Prix Hommage Jacques-Simard/René-O.-Lachance \\ Rédaction scientifique et rayonnement de la Société de protection des plantes du Québec \\ Claude Richard}

Après l'obtention d'un baccalauréat de la Faculté de foresterie et de géodésie de l'Université Laval, Claude Richard a poursuivi ses études à la maîtrise et au doctorat à cette même université dans le domaine des champignons mycorhiziens de l'épinette noire et en phytopathologie générale. Après une année d'enseignement de la botanique, toujours à la Faculté de foresterie et de géodésie, il a décroché un emploi de chercheur au Centre de recherche et de développement sur les sols et les grandes cultures d'Agriculture et Agroalimentaire Canada (AAC) à Québec. II a donc troqué l'épinette noire pour les légumineuses fourragères et fait carrière pendant 21 ans en phytopathologie agricole. Ses travaux ont porté, entre autres, sur différents agents pathogènes de la luzerne, sur la relation des maladies et de la résistance au froid, sur la sélection génétique de la luzerne pour la résistance aux maladies ainsi que sur des agents glaçogènes. Auteur très prolifique, il a à son actif de nombreuses publications scientifiques et de vulgarisation. Il a supervisé les travaux d'étudiants à la maîtrise et au doctorat. II a aussi été actif au sein de comités provinciaux et de sociétés scientifiques, y compris la Société de protection des plantes du Québec (SPPQ) dont il fut le président en 1987-1988.

Le Dr Richard a acquis une renommée enviable dans le travail d'édition scientifique. II a d'abord été rédacteur associé de Phytoprotection, de la Revue canadienne de phytopathologie et du Journal canadien de botanique, puis il a assuré la fonction de rédacteur en chef de Phytoprotection de 1982 à 1985. Ses articles scientifiques publiés dans Phytoprotection de même que son travail soutenu comme rédacteur en chef ont permis à la revue d'acquérir un standard de qualité qui a contribué au rayonnement de la Société. II a aussi dirigé l'édition française du livre Maladies et ravageurs des cultures légumières au Canada et traduit Maladies des grandes cultures au Canada. Sa contribution exceptionnelle en phytopathologie et dans l'édition scientifique a été soulignée par la SPPQ et également par les Sociétés canadienne et américaine de phytopathologie qui l'ont nommé soit membre émérite, soit membre honoraire. On lui a aussi décerné le prix Dr. and Mrs. D.L. Bailey (CPS-

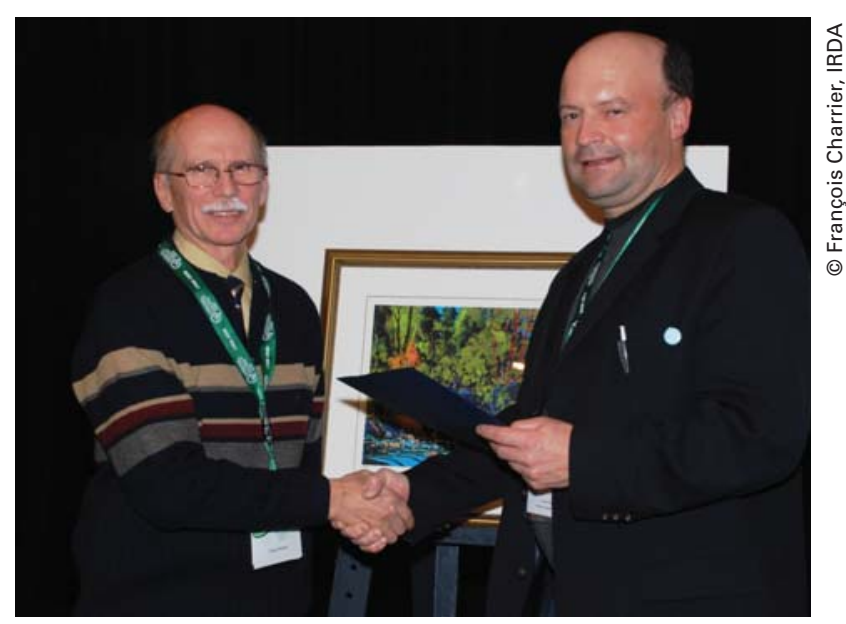

Claude Richard et Danny Rioux

$\mathrm{SCP}$ ) et le prix Agcellence (prix d'excellence dans le secteur agroalimentaire).

À l'occasion de la commémoration de son centenaire (1908-2008), la Société de protection des plantes du Québec est fière de présenter le prix Hommage Jacques-Simard $1 /$ René-O.-Lachance ${ }^{2}$ - Rédaction scientifique et rayonnement au Dr Claude Richard en gage de reconnaissance pour sa contribution inestimable au rayonnement de la revue Phytoprotection et de la Société de protection des plantes du Québec.

Prix Hommage remis par le Dr Danny Rioux, rédacteur en chef de Phytoprotection de 2005 à 2008 et président de la Société en 2003-2004; chercheur au Centre de foresterie des Laurentides (CFL) du Service canadien des forêts (SCF) à Québec; leader de la recherche en pathologie forestière, notamment sur la compréhension des interactions entre l'arbre et les agents pathogènes/insectes à l'aide de la microscopie, d'outils de biochimie ou de biologie moléculaire, sur les risques phytosanitaires, sur les réactions de l'arbre à la suite des stress abiotiques, sur la stimulation des mécanismes de défense, dont le compartimentage, et sur la physiologie de la différenciation du bois.

1. Jacques Simard. Phytopathologiste et premier rédacteur en chef de Phytoprotection de 1962 à 1964.

2. René O. Lachance. Phytopathologiste et président de la Société en 1962-1963, doyen de la Faculté d'agriculture de I'Université Laval de 1967 à 1971, membre honoraire de la Société canadienne de phytopathologie et Commandeur de l'Ordre du Mérite agronomique de l'Ordre des agronomes du Québec.

"He (R.O. Lachance) [...] spearheaded the establishment of Phytoprotection as the official journal of the Society." Sackston, W.E. 1992. René-O. Lachance, 1909-1992. Can. J. Plant Pathol. 14: 115. 


\section{Prix Hommage Henri-Généreux \\ Pionnier - Chercheur administrateur \\ Gilbert Banville}

«Visionnaire [...] Ses recherches ont contribué à changer le portrait de la production de la pomme de terre au Québec quant à la rigueur de la régie, à la qualité des semences, au choix des variétés et à la prévention des maladies."

Ordre des agronomes du Québec $70^{\mathrm{e}}$ anniversaire (2007)

Bachelier en sciences agronomiques de I'Université Laval, Gilbert Banville a obtenu une maîtrise en pathologie végétale de I'University of Maine pour des travaux sur le Rhizoctonia solani et un doctorat de I'University of lowa pour sa thèse sur le développement du périthèce du Ceratocystis fimbriata. Après un stage en virologie à I'Institut National de Recherche Agronomique (INRA) de Versailles en 1969, il a été à l'emploi du ministère de l'Agriculture du Québec, dont il a dirigé la Station de recherche Les Buissons jusqu'à sa retraite en 1996. Agronome de terrain avant tout, il a mené ses recherches en production et en protection des plantes, surtout la pomme de terre. Il a élucidé les mécanismes de base du complexe Rhizoctonia solani, notions qui ont modifié les approches de protection de cette culture au Québec et qui ont valu au Dr Banville une notoriété internationale. II a aussi préparé l'implantation d'un programme d'amélioration génétique qui a généré jusqu'ici une douzaine de variétés de pommes de terre, les premières à voir le jour au Québec. II est l'auteur de nombreux ouvrages scientifiques et a été plusieurs fois conférencier invité au Canada et aux États-Unis. II fut le premier francophone élu au Bureau de direction de la Potato Association of America. Il a présidé I'organisation du $74^{\mathrm{e}}$ Congrès de cette organisation, tenu pour la première fois au Québec. Le Dr Banville est membre de la SPPQ depuis 50 ans. II en fut président en 1984-1985, période durant laquelle il a mené à bien l'incorporation de la Société. II a participé à plusieurs comités et fut rédacteur associé de Phytoprotection.

Les réalisations scientifiques et administratives du Dr Banville ont été plus d'une fois reconnues. L'Ordre des agronomes du Québec lui a décerné la Médaille de distinction agronomique en 1987 et le titre de Commandeur de l'Ordre du Mérite agronomique en 2007. La Médaille commémorative du $125^{\mathrm{e}}$ anniversaire de la Confédération du Canada lui a été remise

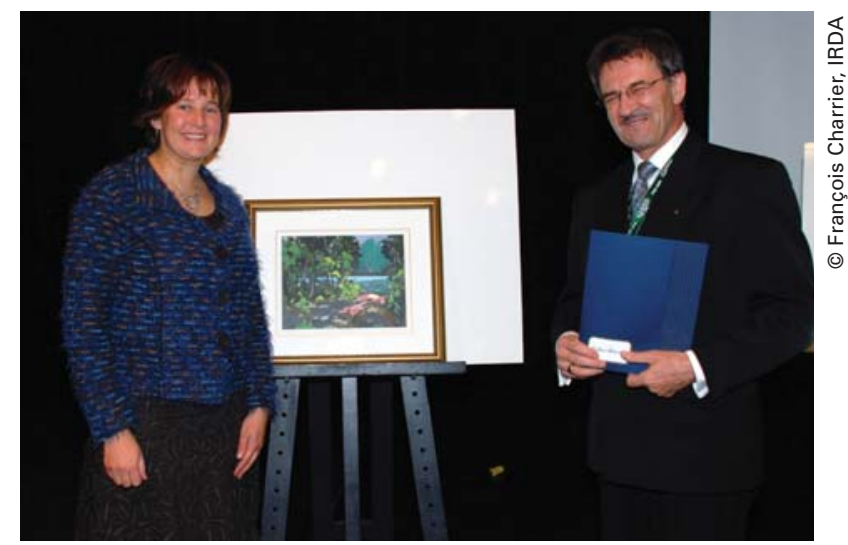

Vicky Toussaint et Gilbert Banville

en 1990 puis, en 1997, le Gouvernement du Québec I'a nommé Commandeur de l'Ordre du Mérite agricole du Québec avec la mention "Très grand mérite spécial ". II est fier de reconnaître qu'au début de sa carrière, c'est $M$. Henri Généreux, de beaucoup son aîné et plus tard un grand ami personnel, qui I'a introduit dans les milieux scientifiques du domaine de la pomme de terre et de la protection des plantes au Canada et aux États-Unis.

En cette Année internationale de la pomme de terre (http://www.potato2008.org/fr/index.html) et à l'occasion de la commémoration de son centenaire (19082008), la Société de protection des plantes du Québec est fière de présenter le prix Hommage HenriGénéreux', Pionnier - Chercheur administrateur au Dr Gilbert Banville en gage de reconnaissance pour sa contribution inestimable aux connaissances sur la protection de la pomme de terre et au rayonnement de la Société de protection des plantes du Québec.

Prix Hommage remis par la Dre Vicky Toussaint, registraire de la Société et présidente en 2004-2005, chercheure au Centre de recherche et de développement en horticulture d'Agriculture et Agroalimentaire Canada $(A A C)$ à Saint-Jean-sur-Richelieu; pionnière et leader de la recherche en phytopathologie agricole, notamment sur la lutte intégrée aux maladies bactériennes et aux pathologies postrécoltes, sur l'écologie microbienne et l'épidémiologie qualitative et quantitative des agents pathogènes des cultures horticoles.

1. Henri Généreux. Phytopathologiste et président de la Société en 1966-1967; nommé membre honoraire de la Potato Association of America (PAA) en 1992.

"Henri Généreux was destined to become a highly respected plant pathologist, and an authority in potato diseases." Estey, R.H. 1994. Essays on the Early History of Plant Pathology and Mycology in Canada. McGill-Queen's University Press, Montréal, Canada. $384 \mathrm{p}$.

"Sa contribution la plus remarquable en pathologie de la pomme de terre consiste en ses recherches et publications sur le flétrissement bactérien (Clavibacter michiganensis ssp. sepedonicus). " Boulanger Généreux, S. 2003.

[http://www.umaine.edu/paa/paa\%20insider2003-3_jul.pdf]. 


\section{Hommage William-Lochhead}

\section{Pionnière et Force vive en malherbologie}

\section{Anne Légère}

Après l'obtention d'un baccalauréat de I'Université Laval et d'une maîtrise en biologie végétale de cette même université, Anne Légère a rempli différents contrats de recherche et d'enseignement avant d'être engagée comme chercheure en malherbologie au Centre de recherches et de développement sur les sols et les grandes cultures d'Agriculture et Agroalimentaire Canada ( $A A C$ ) à Québec. Sentant le besoin de parfaire ses connaissances dans le domaine, elle a poursuivi ses études à Purdue University en Indiana. En 1986, avec un doctorat en poche, elle a réintégré son poste de chercheure à $A A C$ à Québec. Elle a par la suite travaillé quelques années au Plant Science Department du Collège Macdonald de I'Université McGill comme professeure associée invitée, puis comme chercheure au Centre de recherches d'AAC à Saskatoon, avant de revenir à Québec. Huit ans plus tard, elle est retournée à Saskatoon où elle travaille encore aujourd'hui. La Dre Légère a été professeure associée à I'Université Laval, à I'Université McGill et à I'University of Saskatchewan.

Les activités de recherche de la Dre Légère ont porté principalement sur l'impact agro-écologique des pratiques culturales sur les communautés de plantes adventices, surtout en grandes cultures. Plus récemment, ses efforts se sont concentrés sur les cultures transgéniques, évaluant le flux génétique et l'introgression de transgènes dans les plantes adventices sauvages. Elle s'intéresse également à la biodiversité des écosystèmes agricoles et aux relations des plantes adventices avec d'autres taxons. La Dre Légère a à son actif de nombreuses publications scientifiques. Elle a également été active au sein de plusieurs sociétés scientifiques. Elle a été directrice des publications, directrice intérimaire à l'éducation et a fait partie du conseil d'administration et de divers comités de la Weed Science Society of America. Son travail au sein de cette Société lui a valu, en 2001, la nomination de "Fellow". Elle a aussi été présidente de la Société canadienne de malherbologie en 2006. Elle a participé à l'organisation de nombreux congrès, dont à deux reprises le congrès international de malherbologie, celui du Brésil en 2000 et celui du Canada en 2008. Elle a aussi siégé à plusieurs comités d'organismes subventionnaires canadiens (FCAR-FQRNT, CORPAO et CRSNG) et étrangers. La Dre Légère est

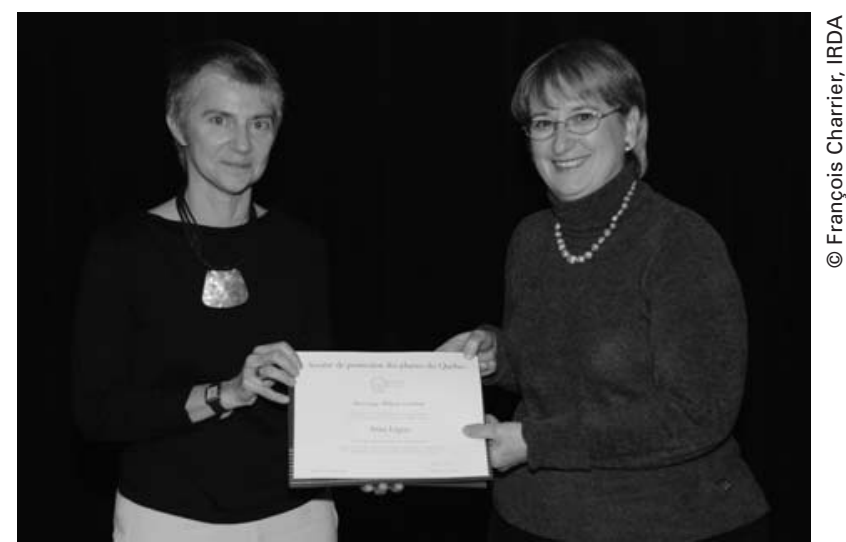

Anne Légère et Diane Benoit

membre de la Société de protection des plantes du Québec depuis 18 ans. Elle a été la première - et à ce jour la seule - femme rédactrice en chef de la revue Phytoprotection et elle a relevé ce défi avec brio pendant quatre ans, soit de 1991 à 1995. Elle a été I'un des évaluateurs des dossiers de candidature à la bourse du centenaire et à la bourse étudiante de la SPPQ en 2008.

À l'occasion de la commémoration de son centenaire (1908-2008), la Société de protection des plantes du Québec est heureuse de présenter I'hommage William-Lochhead ${ }^{1}$ - Pionnière et Force vive en malherbologie au Dre Anne Légère en gage de reconnaissance pour sa contribution inestimable au rayonnement de la malherbologie, de la revue Phytoprotection et de la Société.

Hommage remis par la Dre Diane Benoit, rédactrice technique et rédactrice associée de Phytoprotection; chercheure au Centre de recherche et de développement en horticulture d'Agriculture et Agroalimentaire Canada (AAC) à Saint-Jean-sur-Richelieu; pionnière et leader de la recherche sur l'écologie des populations d'herbe à poux, sur la dynamique et la modélisation des patrons d'émergence des mauvaises herbes en fonction des systèmes de production et des variations climatiques, sur les rotations et le sarclage mécanique en cultures horticoles.

1. William Lochhead (1864-1927). Fondateur de la Société et président de 1908 à 1925.

"Somewhat more of an entomologist than a phytopathologist" (Estey 2007), "William Lochhead founded and became a long time president of the Québec Society for the Protection of Plants, the oldest such society in the world to be continuously functioning under its original name" (Estey 1994). William Lochhead et Douglas Weir, premier secrétaire-trésorier de la Société, ont fait le point sur le problème des mauvaises herbes au Québec. En 1910, le professeur Lochhead a lancé un cri d'alarme: "Pour la province de Québec, il n'est pas actuellement de problème agricole plus ardu que celui des mauvaises herbes " (Émond 1970).

Émond, G. 1970. L'histoire et les perspectives d'avenir de la lutte aux mauvaises herbes au Québec. Phytoprotection 51: 134141.

Estey, R.H. 1994. Essays on the early History of Plant Pathology and Mycology in Canada. McGill-Queen's University Press, Montréal, Canada. 384 p.

Estey, R.H. 2007. 150 years of teaching plant pathology at McGill University. Phytoprotection 88: 1-7. 


\section{Hommage James-Fletcher}

\section{Pionnier et Force vive de l'entomologie Conrad Cloutier}

«... son dévouement à la formation des étudiants [...]. II n'hésite pas à leur offrir sa disponibilité et son support absolu [...]. Il figure parmi les grands entomologistes contemporains du Québec."

\section{Jacques Brodeur et Michel Cusson (2006)}

Après avoir complété baccalauréat et maîtrise (M.Sc.) en biologie à I'Université Laval, Conrad Cloutier a obtenu un doctorat (Ph.D.) de Simon Fraser University (1978), sa thèse portant sur l'écologie nutritionnelle des parasitoïdes de pucerons. Professeur au Département de biologie de la Faculté des sciences et génie (FSG) de I'Université Laval depuis 1977, il enseigne les bases de l'entomologie aux étudiants des programmes de biologie et d'agronomie (Faculté des sciences de l'agriculture et de l'alimentation FSAA). Aux $2^{\mathrm{e}}$ et $3^{\mathrm{e}}$ cycles, il enseigne le contrôle naturel des insectes et la lutte biologique. II a supervisé de nombreux étudiants chercheurs des programmes de biologie de la FSG et de biologie végétale de la FSAA. Parmi ses étudiants, on trouve aujourd'hui des professeurs, des chercheurs scientifiques à Agriculture et Agroalimentaire Canada (AAC) et au Service canadien des forêts (SCF), des entomologistes et des biologistes professionnels de centres de recherche et de services publics québécois.

Les recherches du Dr Cloutier concernent les relations des plantes avec les insectes herbivores et, au niveau trophique supérieur, avec les insectes entomophages comme principaux ennemis naturels des insectes herbivores. Ses travaux ont porté notamment sur la lutte au doryphore de la pomme de terre par des insectes prédateurs, en particulier la punaise pentatomide Perillus bioculatus. Toujours chez la pomme de terre et les insectes associés comme système modèle, il a examiné les effets non intentionnels de la résistance des plantes chez les insectes non ciblés, en particulier la résistance transgénique, en collaboration avec des collègues du Centre de recherche en horticulture. À titre de chercheur principal, le Dr Cloutier a dirigé des travaux d'équipe sur la lutte biologique en cultures serricoles contre des ravageurs comme les thrips et sur les effets non intentionnels des interactions directes entre deux ou plusieurs agents utilisés conjointement (concept de

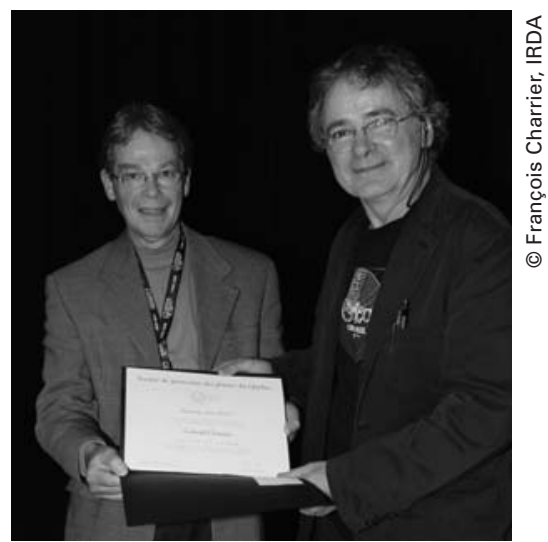

Raymond-Marie Duchesne et Conrad Cloutier

prédation intraguilde). Conrad Cloutier fut I'un des premiers à étudier ce concept, à le mettre en évidence chez les insectes prédateurs et à discuter des conséquences en lutte biologique ${ }^{1}$. Avec des collègues du Centre de foresterie des Laurentides (CFL) et du ministère de I'Agriculture, des Pêcheries et de I'Alimentation du Québec (MAPAQ), Conrad Cloutier a aussi contribué au développement des connaissances de l'écologie et de la biologie des ravageurs du sapin baumier cultivé comme arbre de Noël dans le but de développer la lutte raisonnée et le dépistage dans cette filière agroforestière où l'usage d'insecticides et d'acaricides est traditionnellement I'approche de lutte principale, sinon exclusive. En collaboration avec ses étudiants et des collègues de I'Université Laval, les travaux actuels du Dr Cloutier concernent la résistance des insectes aux parasitoïdes, par association avec des symbiotes bactériens, et leurs réponses à la température et aux autres stress environnementaux étudiées par une approche protéomique. Rédacteur associé de Phytoprotection et membre de la Société de protection des plantes du Québec (SPPQ), le Dr Cloutier, en collaboration avec des étudiants et des collègues, a contribué très activement aux assemblées et aux ateliers de communications scientifiques des réunions annuelles de la Société. En 2005, la Société d'entomologie du Québec lui a décerné la Distinction entomologique.

1. Brodeur, J. et Cusson, M. 2006. Antennae 13 (1): 12-13.

2. James Fletcher (1852-1908). Membre fondateur de la Société, James Fletcher était entomologiste avant tout, mais aussi botaniste et phytopathologiste. II était considéré comme une autorité de par ses connaissances hors du commun de I'entomologie et de la botanique. Il fut nommé "Fellow» de la Royal Society of Canada et de la Linnean Society of London. "The first entomologist to be employed by the Dominion Department of Agriculture. The most noteworthy of the pioneer plant pathologists in Canada" (Estey 1983, 1994).

Estey, R.H. 1983. A history of the Québec Society for the Protection of plants - Histoire de la Société de protection des plantes du Québec. Phytoprotection 64: 1-22.

Estey, R.H. 1994. Essays on the Early History of Plant Pathology and Mycology in Canada. McGill-Queen's University Press, Montréal, Canada. 384 p. 
À l'occasion de la commémoration de son centenaire (1908-2008), la Société de protection des plantes du Québec est fière de présenter I'hommage JamesFletcher $^{2}$ - Pionnier et Force vive de I'entomologie au Dr Conrad Cloutier en gage de reconnaissance pour sa contribution remarquable au rayonnement de I'entomologie, de la revue Phytoprotection et de la Société.

Hommage remis par le Dr Raymond-Marie Duchesne, président de la Société en 1986-1987; leader de la recherche en entomologie, notamment sur I'hespérie des graminées; leader de la mise en œuvre de la Stratégie phytosanitaire du ministère de I'Agriculture, des Pêcheries et de I'Alimentation du Québec, de la recherche sur le B.t., de la lutte physique et de la lutte intégrée au doryphore de la pomme de terre. On lui reconnaît de grands talents de vulgarisateur scientifique auprès des étudiants et du grand public. On lui doit, ainsi qu'au Dr Jean-Marie Perron, la création de la Maison des Insectes à Québec.

\section{Hommage Carrie-Derick \\ Pionnière et Force vive de la phytopathologie agricole \\ Carole Beaulieu}

Après avoir complété un baccalauréat en biologie à I'Université du Québec à Rimouski, Carole Beaulieu a poursuivi ses études à la maîtrise et au doctorat en biologie végétale à I'Université Laval. Elle a par la suite effectué un stage postdoctoral à I'Université libre de Bruxelles en biologie moléculaire, puis à I'University of Florida en phytopathologie. Elle est maintenant professeure titulaire au Département de biologie de I'Université de Sherbrooke. Elle y enseigne la phytopathologie depuis 18 ans. Elle a été la première à I'Université de Sherbrooke à offrir un cours dédié aux interactions plantes micro-organismes. Dre Beaulieu a également été professeure invitée à I'University of Idaho pendant un an. Elle a dirigé et codirigé plus d'une quarantaine d'étudiants aux cycles supérieurs. L'Association générale des étudiants et étudiantes en sciences de I'Université de Sherbrooke lui a décerné le Prix de reconnaissance pour la qualité de son enseignement. Carole Beaulieu et ses étudiants participent régulièrement aux activités de la Société de protection des plantes du Québec (SPPQ). Elle a d'ailleurs présidé à deux reprises (1995 et 2004) I'organisation de la Réunion annuelle de la SPPO. Carole Beaulieu a aussi contribué à la $4^{\mathrm{e}}$ édition (2003) du livre Noms des maladies des plantes au Canada publié par la Société.

Les travaux de recherche de Dre Beaulieu portent sur les interactions plantes hôtes-agents pathogènes. Elle étudie entre autres le modèle Streptomyces scabies-pomme de terre et s'intéresse aussi à la lutte biologique et à la caractérisation microbienne de différents substrats. De nombreuses publications scientifiques sont issues de ses recherches et collaborations. Carole Beaulieu a été codirectrice du Regroupement de recherche en productivité végétale, ce qui l'a conduite à la direction du Centre Sève, un centre de recherche en amélioration végétale regroupant 50 chercheurs du Québec. Elle est également représentante nord-américaine du réseau Biotechnologies végétales de I'Agence universitaire de la francophonie. Elle a aussi participé à plusieurs comités scientifiques de I'ACFAS, du FCAR-FORNT, du CORPAQ et du CRSNG.

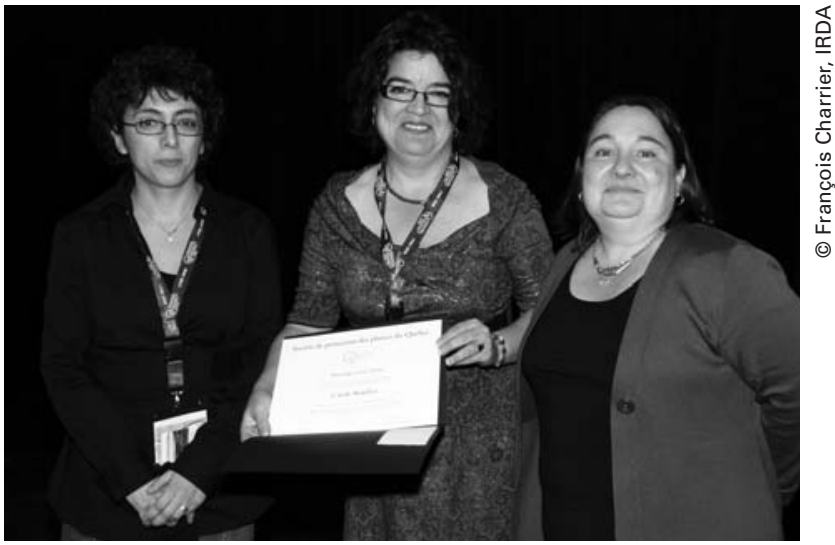

Anne-Marie Simao-Beaunoir, Carole Beaulieu et Odile Carisse

Carole Beaulieu nous a avoué être surprise et très flattée d'être associée au nom de Carrie Derick. À des époques différentes, elles ont réalisé des recherches et enseignements sur la tumeur du collet (Agrobacterium tumefaciens). Toutes deux ont élu domicile dans les Cantons de I'Est et partagent une humanité admirable et une détermination hors du commun.

À l'occasion de la commémoration de son centenaire (1908-2008), la Société de protection des plantes du Québec est heureuse de présenter I'hommage Carrie-Derick $^{1}$ - Pionnière et Force vive de la phytopathologie agricole au Dre Carole Beaulieu en gage de reconnaissance pour sa contribution remarquable au rayonnement de la phytopathologie et de la Société.

Hommage remis par la Dre Anne-Marie SimaoBeaunoir, collègue de la Dre Carole Beaulieu et adjointe administrative du Centre Sève; directrice de la Société de protection des plantes du Québec, webmestre et membre du comité des bourses étudiantes de la Société; et par la Dre Odile Carisse, présidente de la Société en 2000-2001; chercheure au Centre de recherche et de développement en horticulture d'Agriculture et Agroalimentaire Canada 
(AAC) à Saint-Jean-sur-Richelieu; pionnière et leader de la recherche et du développement en phytopathologie agricole, notamment de l'épidémiologie et des systèmes prévisionnels, du dépistage et de la lutte raisonnée aux maladies des plantes horticoles.

1. Carrie Derick (1862-1941). Née à Clarenceville, dans les Cantons de I'Est, Carrie Derick "a milité pour l'avancement des femmes dans les domaines de l'éducation et des professions, pour l'amélioration des conditions sociales et pour le droit de vote des femmes. " http://www.mta.ca/faculty/arts/canadian_studies/francais/realites/guide/canadiennes/index.html

"Carrie Derick se mérite la Logan Gold Medal en sciences naturelles et obtient son baccalauréat B.A. en 1890 à McGill University. En septembre 1891, elle est la première femme enseignante à McGill. En 1911-1912, pionnière à titre de première professeure d'université au Canada, Carrie Derick présenta dans le cadre de "Somerville lecture" un exposé intitulé: "Diseases in plants with special reference to crown gall (tumeur du collet) ". [Coïncidence! La tumeur du collet était le sujet des thèses de maîtrise et de doctorat de Carole Beaulieu.] Carrie Derick a été "thesis adviser " de Douglas Weir, premier secrétairetrésorier de la Société. Weir était étudiant de W. Lochhead, fondateur de la Société. À sa retraite en 1929, Carrie Derick devint la première femme professeur émérite à McGill». Estey, R.H. 1994. Essays on the Early History of Plant Pathology and Mycology in Canada. McGill-Queen's University Press, Montréal, Canada. 384 p.

\section{Hommage Rodolphe-O.-Paradis/Claude-Ritchot Pionnier et Force vive de l'entomologie agricole Guy Boivin}

"Ses pairs reconnaissent sa contribution remarquable à l'entomologie, ainsi que son dévouement auprès des étudiants. Guy Boivin est un ambassadeur exceptionnel pour la recherche et pour le Québec."

Jacques Brodeur (2008) ${ }^{1}$

Guy Boivin a complété un baccalauréat et une maîtrise à I'Université de Montréal et a obtenu, en 1981, un doctorat de I'Université McGill. Il fut engagé la même année comme chercheur en entomologie au Centre de recherche et de développement en horticulture d'Agriculture et Agroalimentaire Canada (AAC) à Saint-Jean-sur-Richelieu. II est également professeur associé à I'Université McGill, à I'Université du Québec à Montréal et à I'Université Laval. Le Dr Boivin a également été professeur invité à I'Université de Rennes, en France, et à I'Université Catholique de Louvain, en Belgique. II a jusqu'ici supervisé ou co-supervisé les recherches d'une trentaine d'étudiants aux cycles supérieurs.

Les recherches du Dr Boivin portent sur l'écologie comportementale des parasitoïdes, sur la génétique des populations et sur l'utilisation des parasitoïdes en lutte biologique. II s'intéresse particulièrement aux stratégies de reproduction et à l'optimisation des comportements chez les parasitoïdes des œufs. Son laboratoire compte une douzaine d'assistants de recherche, de techniciens et d'étudiants chercheurs. Le Dr Boivin a publié de très nombreux articles scientifiques et une dizaine de livres ou de chapitres de livres. II a été président de la Société d'entomologie du Québec (SEQ), de la Société d'entomologie du Canada (SEC), secrétaire-général des Conférences internationales francophones d'entomologie (CIFE) et il est présentement coprésident du groupe de travail sur les parasitoïdes des œufs de I'Organisation internationale de lutte biologique et intégrée contre les

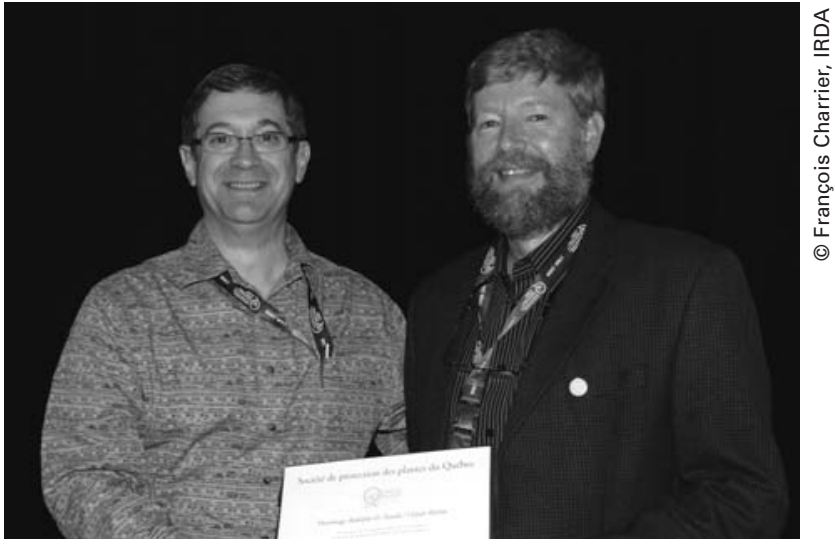

Gaétan Bourgeois et Guy Boivin

animaux et les plantes nuisibles (OILB). Il a également siégé à plusieurs comités du FCAR-FQRNT, du CORPAQ et du CRSNG. Le Dr Boivin s'est admirablement acquitté de la tâche de rédacteur en chef de Phytoprotection de 1985 à 1990 et il fut également rédacteur associé de la revue durant de nombreuses années. II a été I'un des évaluateurs des dossiers de candidature à la bourse du centenaire et à la bourse étudiante de la SPPQ en 2008.

Le Dr Boivin a cofondé le Réseau de lutte intégrée de la Rive-Sud (maintenant PRISME), devenu le plus important service de consultation en lutte intégrée au Québec. On lui a décerné la décoration LéonProvancher de la SEO (1985), le prix C. Gordon-Hewitt de la SEC (1988), le prix Agcellence d'AAC, le méritas Frédérick-Trudel de la Fédération des producteurs maraîchers du Québec, le prix Science des 5RN aux leaders du développement durable du Gouvernement du Canada, le prix des Producteurs du PRISME, 
comme cofondateur du PRISME, et la Distinction entomologique de la SEQ (2007). II a également été nommé "Fellow" de la Société d'entomologie du Canada (1998).

À l'occasion de la commémoration de son centenaire (1908-2008), la Société de protection des plantes du Québec est heureuse de présenter I'hommage Rodolphe-O.-Paradis ${ }^{2} / \mathrm{Claude}$ Ritchot $^{3}$ - Pionnier et Force vive de I'entomologie agricole au Dr Guy Boivin en gage de reconnaissance pour sa contribution inestimable au rayonnement de l'entomologie agricole, de la revue Phytoprotection et de la Société.
Hommage remis par le Dr Gaétan Bourgeois, collègue du Dr Boivin, président de la Société en 20062007 et trésorier de 1995 à 2005; leader de la recherche sur l'impact de la variabilité climatique sur les cultures et leurs bioagresseurs, sur la conceptualisation et la mise au point de modèles bioclimatiques pour la protection et la régie des cultures, sur la prédiction de la phénologie des cultures et des mauvaises herbes, sur la prévision des maladies foliaires et des insectes et sur le développement d'outils informatiques d'aide à la décision.

1. Brodeur, J. 2008. Distinction entomologique. Guy Boivin, chercheur de renommée internationale. Antennae 15 (1): 10. http://www.seq.qc.ca/antennae/archives/Antennae_vol15_no1.pdf

2. Rodolphe-O. Paradis. Entomologiste; président de la Société en 1965-1966 et membre honoraire de la Société et de la SEQ; président de la SEQ en 1972-1973; décoration Léon-Provancher (SEQ) (1981); rédacteur en chef des Annales de la Société d'entomologie du Québec (1967-1977) et du Canadian Journal of Plant Science (1970-1973); "Fellow " de la Société d'entomologie du Canada (1975).

3. Claude Ritchot. Entomologiste; président de la Société en 1977-1978; membre honoraire de la Société et de la SEQ; Commandeur de I'Ordre du Mérite agronomique (Ordre des agronomes du Québec) (1990).

\section{Hommage René-Pomerleau \\ Pionnier et Force vive de la pathologie forestière et moléculaire Louis Bernier, Richard Hamelin et André Lévesque}

\section{Louis Bernier}

“Dr. Bernier is an inspiration for new and established forest pathologists alike and is one of the most visible and recognized forest pathologists in Canada."

The Canadian Phytopathological Society (2002)

Louis Bernier est bachelier en sciences forestières de I'Université Laval. II a par la suite obtenu les grades de maîtrise et de doctorat à I'University of Toronto où il s'est spécialisé en pathologie forestière. Après un stage postdoctoral en biologie moléculaire à I'University of Bath (Angleterre), il est revenu au Québec pour entreprendre, en 1990, une carrière de professeur à I'Université Laval. II a rapidement mis en place un programme de recherche en pathologie forestière. Ce programme a tout de suite fait une large place à I'utilisation des techniques génétiques et moléculaires qui étaient jusque-là encore très peu utilisées par les pathologistes forestiers. L'étude des champignons responsables de la maladie hollandaise de I'orme que le Dr Bernier avait entreprise lorsqu'il étudiait à Toronto est devenue et demeure encore aujourd'hui un thème de recherche privilégié. Les travaux exécutés initialement à l'aide d'approches génétiques ponctuelles ont depuis évolué vers des approches à grande échelle permettant une analyse plus globale du génome. En parallèle, le Dr Bernier s'intéresse à l'étude des populations de champignons phytopathogènes, au développement de moyens de lutte biologique ainsi qu'à la biotechnologie des champignons. Au fil des ans, le Dr Bernier a dirigé ou codirigé 15 étudiants à la maîtrise, 12 doctorants et quatre postdoctorants dans ces domaines. II a en outre développé des collaborations fructueuses avec plusieurs collègues au Québec, au Canada et ailleurs dans le monde. II est l'auteur ou le coauteur de nombreux articles de recherche dans des revues internationales. II est régulièrement invité à présenter des conférences à l'étranger.

Le professeur Bernier a été rédacteur associé à la revue Phytoprotection de 1996 à 2002 et s'acquitte d'une tâche similaire à la Revue canadienne de recherche forestière depuis 2000 . Il a été président de la Société de protection des plantes du Québec (SPPQ) en 1997-1998 et a dirigé le Centre de recherche en biologie forestière (CRBF) de 2001 à 2003. II est codirecteur du Réseau Ligniculture Québec depuis 2001. Il a en outre été impliqué dans la gestion du Réseau Biocontrôle de 2001 à 2006. Il a été récipiendaire, en 2002, du prix Gordon J. Green décerné par la Société canadienne de phytopathologie à un jeune chercheur émérite. Désormais "jeune quinquagénaire ", le professeur Bernier est toujours enthousiasmé par la recherche en milieu universitaire et par la formation de la relève scientifique en pathologie forestière. 


\section{Richard Hamelin}

Richard Hamelin a obtenu un baccalauréat en agronomie de I'Université McGill et une maîtrise de Simon Fraser University (Vancouver) dans le programme de gestion des ravageurs. II a par la suite complété un doctorat en phytopathologie à I'University of Kentucky et a obtenu un poste de chercheur post-doctoral au United States Department of Agriculture (USDA) Forest Service, au Mississippi, puis à I'Université Laval, à Québec. En 1992, il a décroché un poste de chercheur en phytopathologie au Centre de foresterie des Laurentides du Service canadien des forêts (SCF) à Québec. Professeur associé à la Faculté de foresterie et de géomatique de I'Université Laval et à la Faculty of Forestry de I'University of British Columbia, il a dirigé et codirigé plusieurs étudiants à la maîtrise et au doctorat.

Le Dr Hamelin a été I'un des premiers chercheurs au Québec à utiliser des outils moléculaires pour étudier la biologie et l'épidémiologie d'agents pathogènes des forêts. II a ainsi contribué à développer le domaine de l'épidémiologie moléculaire. Ses travaux portent, entre autres, sur l'identification et la caractérisation de gènes de pathogénicité et de reconnaissance des hôtes. L'acquisition de telles connaissances permet d'améliorer le suivi et la prévision de la migration de ces agents pathogènes et aide à la prise de décision. II est l'auteur de nombreux articles scientifiques et chapitres de livres et il a été conférencier invité à plusieurs occasions. Le Dr Hamelin a été président de la Société de protection des plantes du Québec en 1998-1999 et de la Société canadienne de phytopathologie, ainsi que rédacteur associé de la Revue canadienne de recherche forestière. II est présentement directeur de la section "Pathologie forestière" de la Revue canadienne de phytopathologie, de même que rédacteur principal de Phytopathology. II a également participé à plusieurs comités, dont le comité de sélection des subventions du CRSNG, les comités d'évaluation d'unités de recherche de I'Institut National de la Recherche Agronomique (INRA) de Nancy et du Centre de Recherche en Agriculture et Développement de Montpellier. II fait présentement partie du comité consultatif de Genome British Columbia en foresterie. On lui a décerné le prix de reconnaissance de I'Agence canadienne d'inspection des aliments, la prime au mérite du SCF et le prix RNCan de Ressources naturelles Canada pour la créativité et l'innovation.

\section{André Lévesque}

“Dr. C. André Lévesque is one of the most successful and influential young plant pathologists in Canada today. [He] has become a world authority on the molecular taxonomy and diagnostics of Pythium."

The Canadian Phytopathological Society (1999)

André Lévesque détient un baccalauréat en agronomie de I'Université McGill, une maîtrise professionnelle en lutte intégrée de Simon Fraser University, ainsi qu'un doctorat de cette même université. En 1993, le Dr Lévesque a été engagé à Agriculture et Agroalimentaire Canada (AAC). II a occupé un poste de chercheur en phytopathologie au
Centre de recherches agroalimentaires du Pacifique en Colombie-Britannique, d'abord à la station de Vancouver, puis à la station de Summerland. En I'an 2000, il s'est rapproché du Québec en rejoignant l'équipe de biosystématique du Centre de recherches de I'Est sur les céréales et les oléagineux à Ottawa comme spécialiste des champignons produisant des zoospores (Oomycètes et Chytridiomycètes).

Son travail de recherche au doctorat a mené à des publications qui sont encore aujourd'hui des références importantes pour les études d'impact du glyphosate sur les maladies racinaires et la flore fongique du sol. Mentionnons qu'il utilisait déjà à ce moment-là (fin des années 1980) diverses techniques d'ADN recombinant pour étudier les populations de Pythium. Dans le cadre de ses emplois, il a développé le premier système de détection par biopuces en phytopathologie et en mycologie, approche qui a été adaptée par la suite pour la bactériologie, la nématologie et la virologie et qui est maintenant utilisée commercialement au Canada et en Europe. II a publié une phylogénie complète des espèces de Pythium qui est devenue la principale référence moléculaire pour la taxinomie de ce genre important en phytopathologie. II a été le premier à développer, pour l'écologie moléculaire, une biopuce pour un genre complet d'oomycète, soit le genre Pythium qui compte plus de 100 espèces. Le Dr Lévesque travaille en étroite collaboration avec plusieurs laboratoires au Canada, aux États-Unis et en Europe pour développer de meilleurs outils de génomique et de bioinformatique pour le dépistage d'agents phytopathogènes de quarantaine à haut risque, tant en agriculture qu'en foresterie. II codirige une initiative nationale pour la création d'un organisme qui encadrerait, appuierait et aiderait à développer les principales souchothèques canadiennes.

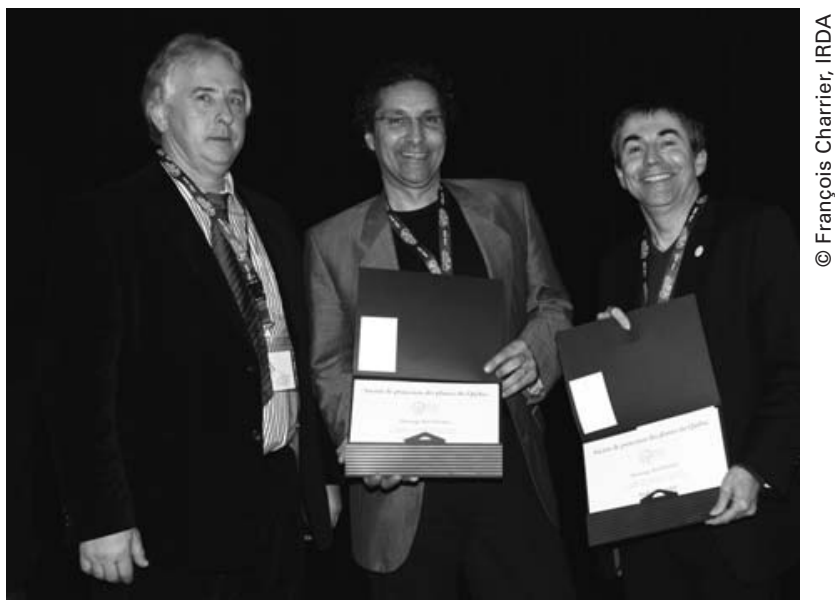

Guy Bussières, Richard Hamelin et André Lévesque

Le Dr Lévesque est professeur associé à I'Université Carleton. II est l'auteur ou le coauteur de nombreux articles scientifiques, incluant deux invitations pour I'Annual Review of Phytopathology. II donne régulièrement des conférences sur invitation, tant au Canada qu'à I'étranger. Le Dr Lévesque a été président de la Société canadienne de phytopathologie (SCP) en 2005-2006. Il a organisé de nombreux collo- 
ques, dont le congrès du $75^{\mathrm{e}}$ anniversaire de la SCP en 2004. Il a fait partie de plusieurs comités au sein de diverses sociétés scientifiques. II a été I'un des évaluateurs des dossiers de candidature à la bourse du centenaire et à la bourse étudiante de la SPPQ en 2008. Le Dr Lévesque a été honoré à plusieurs reprises. II a reçu, entre autres, le prix du jeune chercheur émérite Gordon J. Green de la SCP en 1999.

À l'occasion de la commémoration de son centenaire (1908-2008), la Société de protection des plantes du Québec est fière de présenter I'hommage RenéPomerleau ${ }^{1}$ - Pionnier et Force vive de la pathologie forestière et moléculaire aux chercheurs Louis
Bernier, Richard Hamelin et André Lévesque en gage de sa plus profonde gratitude pour leur contribution inestimable au rayonnement de la mycologie, de la pathologie forestière et moléculaire et de la Société.

Hommage remis par monsieur Guy Bussières, président de la Société durant deux mandats, en 19931994 et 1994-1995; chercheur et enseignant à la Faculté de foresterie, de géographie et de géomatique de l'Université Laval; spécialiste de la classification des arbres, de la foresterie urbaine et de la pathologie forestière.

1. René Pomerleau (1904-1993). Membre de la Société dès 1927 et président de la Société en 1958-1959. Pionnier de la mycologie et de la pathologie forestière au Québec et au Canada. Récipiendaire de très nombreux prix et décorations, notamment: Membre de la Société Royale du Canada (1948), Médaille Parizeau de I'ACFAS (1955), Officier de l'Ordre du Canada (1970), Lauréat du prix Marie-Victorin (1981) et Chevalier de I'Ordre du Québec (1988). Laflamme, G. 1994. René Pomerleau, 1904-1993, 65 ans de carrière. Can. J. Plant Pathol. 16: 253-254.

\section{Hommage William-P.-Fraser/Alain-Asselin Pionnier et Force vive de la phytopathologie agricole Richard Bélanger}

The Powdery Mildews: A Comprehensive Treatise par R.R. Bélanger, W.R. Bushnell, A.J. Dik and T.L.W. Carver. "This is certainly the most detailed and authoritative overview of these fungi yet to have appeared, especially with regard to species on crop plants. All chapters are well-referenced and illustrated, and this text will surely be the vade mecum of those having to confront powdery mildew diseases anywhere in the world for many years to come."

Mycological Research

Richard Bélanger a complété un baccalauréat en sciences forestières à I'Université Laval et a obtenu un doctorat en phytopathologie de State University of New York (SUNY) à Syracuse en 1988. Professeur au Département de phytologie de la Faculté des sciences de I'agriculture et de I'alimentation (FSAA) de I'Université Laval et chercheur au Centre de recherche en horticulture $(\mathrm{CRH})$, le Dr Bélanger est titulaire de la Chaire de recherche du Canada en phytoprotection (2001). II enseigne la phytopathologie et les principes de la lutte intégrée. II est vivement apprécié par ses étudiants pour la qualité et le dynamisme de ses cours et sa grande disponibilité. L'intégration, les progrès et les réalisations des étudiants chercheurs du Dr Bélanger reposent en outre sur la personnalité et les compétences remarquables de Mme Caroline Labbé, professionnelle de recherche. Le laboratoire du Dr Bélanger lui doit cet environnement de recherches diversifiées empreint de collaboration et de défis qui stimule la créativité des étudiants du premier cycle ou des cycles supérieurs.

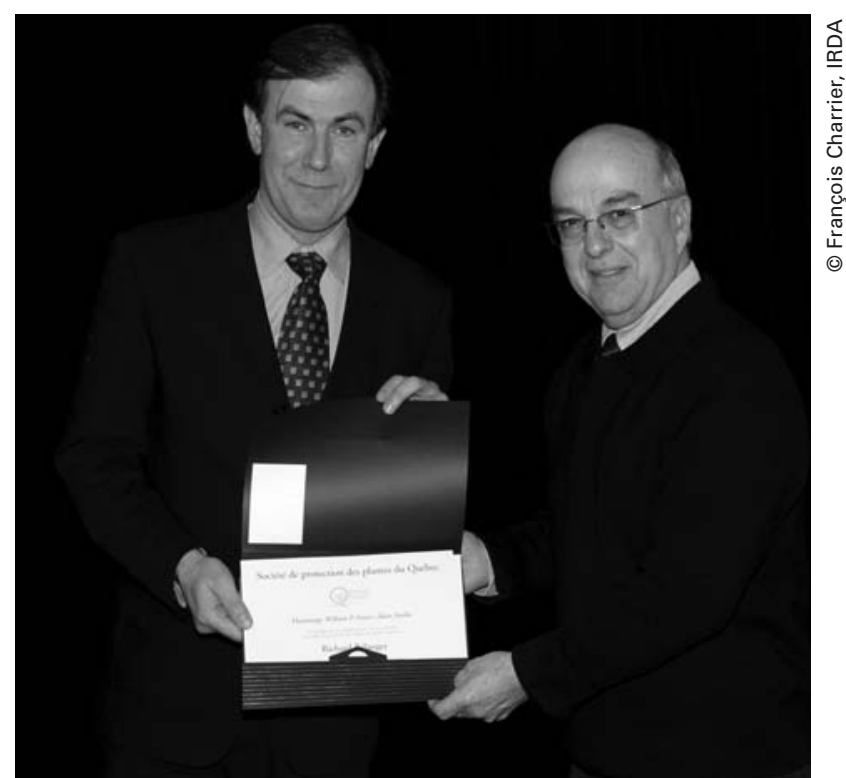

Richard Bélanger et Alain Asselin

Le Dr Bélanger est une autorité en ce qui concerne la lutte biologique aux maladies des cultures serricoles. Ses étudiants chercheurs et collaborateurs ont contribué aux connaissances sur l'écologie et le mode d'action d'agents de lutte biologique et à leur mise en œuvre. Ce leadership a résulté, entre autres, en la publication d'une synthèse produite par T.C. Paulitz et R.R. Bélanger (2001) dans I'Annual Review of Phytopathology. Ses recherches sur la lutte sans 
pesticide au blanc (Erysiphales), telles la lutte biologique et la résistance induite, lui ont valu une reconnaissance internationale et ont résulté en la publication du livre The Powdery Mildews: A Comprehensive Treatise en 2002. La citation de Mycological Research est éloquente à cet égard. On doit au Dr Bélanger, à ses étudiants et à ses collaborateurs le développement du Sporodex®, un biofongicide contre le blanc dont l'activité repose sur I'antagoniste Pseudozyma flocculosa. Fruit de 15 ans d'efforts soutenus en partenariat avec Plant Products Co. Ltée, Sporodex® est le premier produit biologique canadien de cette nature à être homologué au Canada et aux États-Unis. On doit aussi au laboratoire du Dr Bélanger la découverte de la production de phytoalexines comme mécanisme de défense chez le concombre. Son groupe a récemment publié des preuves spatio-temporelles in situ de leur activité. Par ses recherches sur la silice, le groupe du Dr Bélanger a été le premier à promouvoir I'hypothèse que cette dernière agissait par l'induction de réactions de défense. Leurs travaux sur la silice ont été publiés dans les prestigieux Proceedings of the National Academy of Sciences et ont récemment été repris deux fois dans les éditoriaux de Physiological and Molecular Plant Pathology.

À l'image du réseau international de collaborateurs qu'il visite avec son équipe ou accueille dans son laboratoire, le Dr Bélanger a été président de colloques et conférencier invité à de nombreuses reprises au Canada et à l'étranger. Membre du Centre de recherche en horticulture et du Centre de recherche en amélioration végétale (Sève), Richard Bélanger a été président de la Société de protection des plantes du Québec en 1992-1993 et président élu de la Société canadienne de phytopathologie (SCP; 2007-2010). II a été secrétaire, vice-président et président de la division nord-est de I'American Phytopathological Society (APS), en plus d'être élu "Councilor-at-large" de la même Société. II vient également de terminer un mandat au sein du comité du centenaire de l'APS. Le Dr Bélanger a également été le représentant nordaméricain du réseau Biotechnologies végétales de I'Agence universitaire de la francophonie. Le Dr Bélanger est rédacteur associé de la revue Biological Control. II a été récipiendaire, en 1997, du Fisher Scientific Award décerné par la Société canadienne de microbiologie et du prix Gordon J. Green décerné par la SCP à un jeune chercheur émérite. II a aussi reçu en 2004 le prix Outstanding Service Award Councilor-at-large de I'American Phytopathological Society. Lors du congrès portant sur la silice en agriculture tenu en octobre 2008 en Afrique du Sud, on lui a attribué le Prix d'excellence en recherche pour sa contribution exceptionnelle et soutenue aux travaux sur la silice.

À I'occasion de la commémoration de son centenaire, la Société de protection des plantes du Québec est heureuse de présenter I'hommage William-P.Fraser ${ }^{1} /$ Alain-Asselin ${ }^{2}$ - Pionnier et Force vive de la phytopathologie agricole au Dr Richard R. Bélanger en gage de gratitude pour sa contribution remarquable au rayonnement de la phytopathologie et de la Société.

Accompagné du $\mathrm{Dr}$ Asselin, le docteur Russell Tweddell était invité à remettre I'hommage. Le Dr Tweddell est chercheur au Centre de recherche en horticulture (CRH) et professeur au Département de phytologie de la Faculté des sciences de I'agriculture et de I'alimentation (FSAA) de I'Université Laval; président de la Société en 2002-2003 et rédacteur associé de Phytoprotection; leader de la recherche en mycologie, en physiologie des champignons, en phytopathologie (chez la pomme de terre, la carotte et la tomate de serre) et en lutte intégrée, notamment la lutte biologique chez la pomme de terre; il a obtenu le prix Gordon J. Green décerné par la Société canadienne de phytopathologie à un jeune chercheur émérite.

1. William Pollock Fraser (1867-1943). "The first Canadian-born plant pathologist and mycologist to be internationally acclaimed as such" (Estey 1992). Pionnier de la recherche sur le blanc (Erysiphales), il a en outre obtenu un B.A. de Cornell University. Natif de la Nouvelle-Écosse, il a étudié et enseigné dans cette province. II a aussi enseigné au Québec (au Collège Macdonald, où il y a enseigné à Margaret Newton) et en Saskatchewan (professeur émérite du Department of Biology, University of Saskatchewan, Saskatoon) (T.C. Vanterpool 1944, cité par Estey 1992). Margaret Newton et lui sont les pionniers de la recherche sur les races de la rouille noire (Puccinia graminis tritici) chez le blé au Canada. Directeur de la Société de 1916 à 1920, il fut entre autres président de la division canadienne de l'American Phytopathological Society, président de 1931 à 1933, "Charter»" et membre honoraire de la Société canadienne de phytopathologie. Estey, R.H. 1992. William Pollock Fraser (1867-1943): Canadian pioneer plant pathologist-mycologist. Phytoprotection 73: 53-59.

2. Alain Asselin a obtenu un doctorat de Cornell University en 1978. II a été membre actif de la Société et rédacteur associé de Phytoprotection durant plusieurs années, ainsi que professeur de phytopathologie, de virologie et de biochimie végétale et vice-doyen à la FSAA de l'Université Laval. II a été pionnier québécois de la biochimie végétale et des biotechnologies appliquées à la phytopathologie. On doit au Dr Asselin plusieurs avancées fondamentales et appliquées dans les domaines de la virologie, de la phytopathologie et de la biochimie végétale. Nous pouvons citer, notamment, la mise en évidence de protéines virales hôtes-spécifiques; la découverte de nouvelles familles de protéines hydrolytiques, les chitosanases et les glucanases du type de la thaumatine, impliquées dans la défense chez les plantes; le développement d'approches analytiques de laboratoire pour l'étude des protéines associées à la pathogenèse; ou encore ses travaux pionniers en transgénèse végétale sur l'expression de protéines recombinantes d'origine bactérienne à des fins de phytoprotection. Par son enseignement, le Dr Asselin est reconnu parmi les meilleurs professeurs, les plus dynamiques et les plus enthousiastes. II a fait naître la flamme de la recherche chez toute une génération de phytopathologistes et de biochimistes. En 1986, il est devenu le premier récipiendaire québécois du prix Gordon J. Green décerné par la SCP à un jeune chercheur émérite. À l'occasion de la journée commémorative de son centenaire (1908-2008), la Société de protection des plantes du Québec est fière de dédier au Dr Alain Asselin I'hommage Pionnier et Force vive de la phytopathologie agricole. La Société souligne ainsi la contribution remarquable du Dr Asselin au développement de la phytopathologie et au rayonnement de la Société. 


\title{
Hommage Pierre-O.-Thibodeau \\ Réviseur de Noms des maladies des plantes au Canada
}

\author{
Luc Couture et Jean Denis Brisson
}

"Nous tenons à remercier toutes les personnes qui ont contribué à cette nouvelle édition (Noms des maladies des plantes au Canada) et nous leur rendons un témoignage de reconnaissance et d'admiration. Une mention spéciale est adressée à messieurs Luc Couture et Jean Denis Brisson. "

Russell Tweddell et Gilles Émond (2003)

\section{Luc Couture}

Après I'obtention d'un baccalauréat (B.Sc.) en agrobiologie et d'une maîtrise (M.Sc.) en biologie végétale à I'Université Laval, Luc Couture a complété un doctorat (Ph.D.) en 1977 sous la supervision du Dr John C. Sutton de I'University of Guelph sur l'épidémiologie des taches helminthosporiennes de I'orge. Le Dr Couture a été recruté comme chercheur en pathologie des céréales à la Station de recherche d'Agriculture et Agroalimentaire Canada (AAC) à Sainte-Foy. Ses recherches ont porté notamment sur la lutte chimique, la pathologie des semences, les moisissures nivéales et la fusariose de l'épi. Il a collaboré au développement de cultivars de céréales (avoine, orge et blé) avec le Dr Jean-Pierre Dubuc. En 1988, Luc Couture a obtenu une affectation de deux ans et demi au laboratoire du Service de la protection des végétaux du Burkina Faso dans le cadre d'un projet de coopération canadienne; ses travaux étaient concentrés sur le flétrissement bactérien de la tomate. En 1991, il a repris ses activités à la station d'AAC dans le domaine des céréales. À la suite du budget fédéral dévastateur de 1995, la recherche sur les céréales a été pour ainsi dire " effacée " de la station de SainteFoy. Le Dr Couture a dû délaisser les céréales pour se consacrer aux cultures fourragères. Depuis 1996, le Dr Couture s'intéresse donc aux maladies des cultures fourragères, principalement la luzerne, mais aussi aux espèces annuelles comme le maïs. Ses activités sont reliées à la sensibilité variétale aux maladies en général, aux maladies des racines, à la détection des agents pathogènes et à la conservation des fourrages récoltés. Professeur associé de I'Université Laval, le Dr Couture a supervisé et codirigé neuf étudiantes et étudiants aux cycles supérieurs.

Le Dr Couture a participé très activement durant de nombreuses années aux ateliers du comité céréales et du comité plantes fourragères du Conseil des productions végétales du Québec (CPVQ), aujourd'hui devenu le Centre de référence en agriculture et agroalimentaire du Québec (CRAAQ). II a contribué à la rédaction et à l'édition du guide sur les plantes fourragères (CRAAQ 2005) et à celui des céréales de printemps (CPVQ 1988). En 2005, la Société canadienne de phytopathologie lui a décerné le prix Réalisations dans la lutte aux maladies des plantes Achievements in Plant Disease Management Award, prix partagé avec les Drs K.L. Bailey, R.A.A. Morrall, R.K. Gugel et B.D. Gossen pour la rédaction et la publication du livre Maladies des grandes cultures au Canada (2004), une réalisation dont le Dr Couture est particulièrement fier. En plus de sa contribution à la révision scientifique et technique de la $4^{\mathrm{e}}$ édition de Noms des maladies des plantes au Canada (2003),

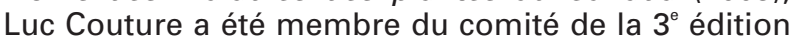
(1992). II a aussi occupé plusieurs fonctions au sein de la Société de protection des plantes du Québec, notamment celles de rédacteur des bulletins de nouvelles et des Échos phytosanitaires de la Société de 1980 à 1983, rédacteur en chef suppléant et rédacteur associé de Phytoprotection, ainsi que rédacteur technique de 1985 à 1988 .

\section{Jean Denis Brisson}

Jean Denis Brisson a complété un baccalauréat (B.Sc.) en agrobiologie et une maîtrise (M.Sc.) en biologie végétale à I'Université Laval, puis un doctorat (Ph.D. en botanique, génétique et systématique) sous la supervision du Dr Larry R. Peterson de I'University of Guelph (1978). Le Dr Brisson a occupé successivement des postes au ministère de I'Agriculture, des Pêcheries et de l'Alimentation du Québec (agronome-chercheur de 1980 à 1996), au Laboratoire de diagnostic en phytoprotection du Québec (entomologiste de 1996 à 1999), puis au Service de la biodiversité et des maladies de la faune du ministère des Ressources naturelles et de la Faune pour des travaux de systématique en entomologie et sur la faune. Jean Denis Brisson est un "phénomène et scientifique hors norme" (Gingras 2005). "Botaniste, entomologiste, microscopiste, virologiste, phytopathologiste et vulgarisateur " (Pierre-O. Thibodeau), le Dr Brisson a à son actif de nombreuses publications scientifiques dans des revues avec comité de lecture dans les domaines de l'entomologie et surtout de la botanique et de la phytopathologie examinés sous les aspects de la taxinomie, de la microscopie électronique et de I'histochimie. II a aussi publié d'assez nombreuses notes taxonomiques et des premières mentions entomologiques. Vulgarisateur, Jean Denis Brisson a produit des textes qui couvrent la botanique (morphologie et physiologie), I'horticulture maraîchère, fruitière et ornementale, I'entomologie ou encore l'ornithologie, en particulier dans les Bulletins de la Société des Amis du Jardin Van den Hende, Fabreries, Option Serre, Phytoma, Phytoprotection et Fleurs, Plantes \& Jardins. Le Dr Brisson est président fondateur (1979) de la Société des Amis du Jardin Van den Hende et fondateur de la librairie Horti-Centre (1985). Membre de très nombreuses sociétés, Jean Denis Brisson a été particulièrement actif à I'Association des entomologistes amateurs du Québec, à la Société d'entomologie du Québec et à la Société de protection des plantes du Québec. Tout comme Luc Couture, en plus de sa contribution à la révision scientifique et technique de la $4^{\mathrm{e}}$ édition de Noms des maladies des plantes au Canada (2003), Jean Denis Brisson a été membre du comité de la $3^{e}$ édition (1992). 
À l'occasion de la commémoration de son centenaire, la Société de protection des plantes du Québec est heureuse de présenter I'hommage Pierre-O.Thibodeau ${ }^{1}$ - Réviseur de Noms des maladies des plantes au Canada aux Drs Luc Couture et Jean Denis Brisson en gage de sa profonde gratitude pour leur contribution remarquable au rayonnement de la protection des plantes et de la Société.

Hommage remis par le Dr Gilles Émond, président du comité du centenaire et lauréat du prix Excellence pour l'ensemble des réalisations. Il fut président du Comité permanent de nomenclature française des maladies des plantes au Canada pendant 15 ans. Durant ses mandats se sont succédé une édition corrigée et deux nouvelles éditions du volume Noms des maladies des plantes au Canada.

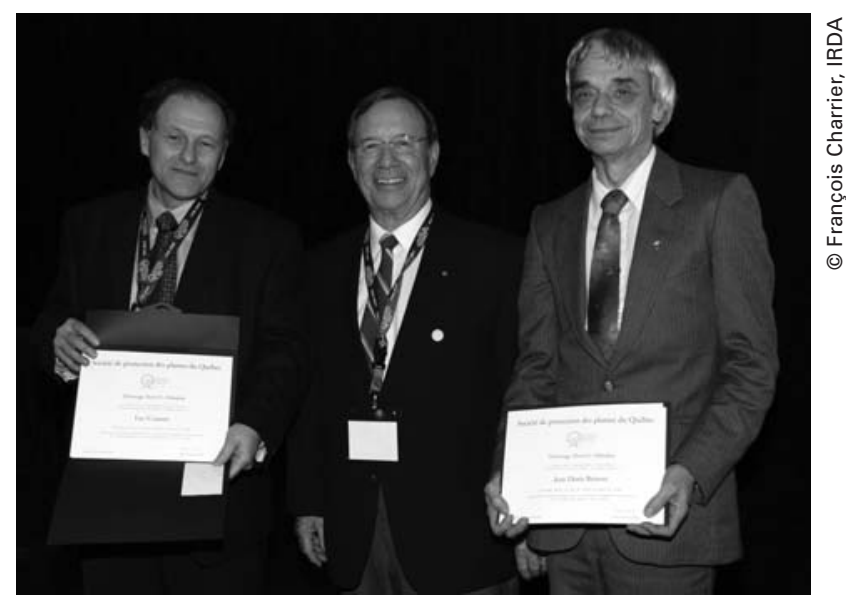

Luc Couture, Gilles Émond et Jean Denis Bisson

1. Pierre-O. Thibodeau (1940-2008). Agronome et chercheur phytopathologiste à l'emploi du ministère de I'Agriculture, des Pêcheries et de I'Alimentation du Québec et de I'IRDA durant plus de 34 ans. Pierre-O. Thibodeau, "l'infatigable, le rassembleur, était un communicateur et un vulgarisateur accompli " (CRAAQ 2002). "Le passionné qui aime donner" (Liette Lambert 2002), "Artisan du Réseau d'avertissements phytosanitaires, son coup de cœur professionnel, tel qu'il le disait lui-même, fut d'être le premier avertisseur du réseau petits fruits, auquel il collabora avec toutes ses énergies durant 25 ans, de 1975 à 1999. Il fut un ardent défenseur de la lutte intégrée et de l'utilisation judicieuse des pesticides " (Réseau d'avertissements phytosanitaires 2008). Membre et président durant plusieurs années du comité petits fruits du CRAAQ et pionnier du diagnostic, on lui doit des travaux originaux sur l'épidémiologie et " la lutte chimique ou biologique contre la pourriture grise (Botrytis cinerea), le développement de stratégies de lutte contre les maladies des légumes de serre et des petits fruits, dont la stèle rouge (Phytophthora fragariae), le développement d'outils de dépistage des ennemis des petits fruits et du programme de certification du fraisier et du framboisier" (Réseau d'avertissements phytosanitaires 2008). En 2002, le CRAAQ lui a décerné le Méritas Frédérick-Trudel pour sa contribution inestimable au développement des cultures en serre et des petits fruits. Pierre-O. Thibodeau a été secrétaire du Comité permanent de nomenclature des $2^{\mathrm{e}}$ et $3^{\mathrm{e}}$ éditions (1975 et 1992) et membre du Comité permanent de nomenclature de la $4^{e}$ édition de la publication Noms des maladies des plantes au Canada.

La $4^{e}$ édition de l'ouvrage Noms des maladies des plantes au Canada a été publiée en 2003. À cette occasion, le Dr Gilles Émond, président du Comité permanent de nomenclature française des maladies des plantes, et le Dr Russell Tweddell, président de la Société (2002-2003), ont témoigné leur reconnaissance au Comité de nomenclature, avec une mention spéciale adressée à messieurs Luc Couture et Jean Denis Brisson, réviseurs de cette $4^{e}$ édition en compagnie du Dr Émond.

Bailey, K.L., L. Couture, B.D. Gossen, R.K. Gugel et R.A.A. Morrall (directeurs de la rédaction). 2004. Maladies des grandes cultures au Canada, $1^{\text {re }}$ éd. Société canadienne de phytopathologie, Saskatoon, Canada. 318 p.

Bélanger, G., L. Couture et G. Tremblay (directeurs de la rédaction). 2005. Les plantes fourragères. Centre de référence en agriculture et agroalimentaire du Québec (CRAAQ), Québec, Canada. 209 p.

Couture, L., J.D. Brisson et G. Émond (réviseurs scientifiques et techniques). 2003. Noms des maladies des plantes au Canada, $4^{e}$ éd. Société de protection des plantes du Québec, Québec, Canada. $340 \mathrm{p}$.

Gingras, D. et collab. 2005. Jean Denis Brisson: un scientifique «hors norme». Antennae 12 (3): 12-14.

\section{Hommage J.-Champlain-Perrault/Elzéar-Campagna}

\section{Services publics en phytoprotection ${ }^{1}$}

\section{Service des relevés et des diagnostics (MRNF) Laboratoire de diagnostic en phytoprotection du Québec (MAPAQ) Réseau d'avertissements phytosanitaires (MAPAQ)}

Service des relevés et des diagnostics, Direction de l'environnement et de la protection des forêts, ministère des Ressources naturelles et de la Faune du Québec (MRNF)
Le laboratoire de diagnostic du Service des relevés et des diagnostics de la Direction de l'environnement et de la protection des forêts, du ministère des Ressources naturelles et de la Faune (MRNF), a une

1. Pour leur contribution inestimable à la préparation de ce texte, les auteurs remercient chaleureusement Mme Louise Innes (pour l'historique du Service des relevés et des diagnostics), MM. Michel Letendre et Gilles Émond (pour l'historique du Réseau d'avertissements phytosanitaires) et MM. Gérard Gilbert et Gilles Émond (pour l'historique du Laboratoire de diagnostic en phytoprotection du Québec). 
longue histoire. Les premiers travaux en pathologie forestière remontent à 1930, alors qu'a débuté la carrière du Dr René Pomerleau, pionnier de la mycologie et de la pathologie forestière au Québec et au Canada, à la pépinière forestière de Berthierville (maintenant Berthier). Il a dirigé des recherches dans les laboratoires de pathologie du ministère des Terres et Forêts (MTF) du Québec de 1930 à 1937 à la pépinière forestière de Berthier, puis, de 1938 à 1952, à Québec.

Du côté de l'entomologie, la création, au niveau provincial, du Service d'entomologie du ministère des Terres et Forêts remonte à 1938, à la suite d'une épidémie spectaculaire du diprion européen de l'épinette. Le Service a alors entrepris l'inventaire des insectes forestiers. L'identification et l'élevage des insectes reçus étaient réalisés à la station de recherches forestières de Duchesnay, où le Service d'entomologie possédait un insectarium comprenant un laboratoire et une chambre d'élevage. Monsieur A.R. Gobeil était responsable de cette section. En 1972, les laboratoires de diagnostic en entomologie et pathologie forestière du Service d'entomologie et de pathologie (SEP) de la Direction de la conservation du MTF ont vu le jour au Complexe scientifique du Québec, rue Einstein, à Sainte-Foy. L'équipe des années 1970 était formée de:

- Jean-Guy Davidson, responsable du laboratoire de pathologie, et de Chantal Lachance, Solange Simard, Claude Paradis, Robert Picher, Denis Guérin, Gaston Laflamme, Guy Croteau, Gérald Mercier, Gaston Déry et Pierre-Émile Rocray en pathologie forestière;

- René Béique, responsable du laboratoire d'entomologie, et Diane Paré, Céline Piché, Lucie Marchand, Lina Breton, Jean-Paul Campeau, M. Cossette et Gilles Bonneau en entomologie forestière.

En 1983, la responsabilité du Relevé des insectes et des maladies des arbres est transférée du gouvernement fédéral au gouvernement provincial. En plus des laboratoires, le SEP possède la Collection de champignons des arbres du Québec (CCAQ) et la Collection d'insectes du Québec (CIQ). L'équipe des laboratoires de diagnostic du SEP du ministère des Ressources naturelles (MRN) des années 1980 était composée de:

- Louise Innes, Solange Simard (CCAQ), Manon Gignac, Robert Picher et Paul Laflamme en pathologie;

- Gilles Bonneau, Lucie Marchand, Céline Piché (CIO), Diane Paré, Lina Breton, Denis Lévesque, Lynda Simard et Danièle Pouliot en entomologie.

Le personnel du relevé, des laboratoires et de la CCAO est demeuré au Complexe scientifique jusqu'en 1986. Ce personnel ainsi que les équipements des laboratoires et de la CCAQ du Service de la protection contre les insectes et les maladies (SPIM) sont déménagés au 1283, boulevard Charest Ouest, à Québec. Ils y sont demeurés pendant 15 ans. Puis, en 2001, ils sont retournés au Complexe scientifique de Québec, à la suite d'un court séjour à l'Atrium.
En 2008, on ne parle désormais plus que d'un seul laboratoire qui s'occupe également de l'entomologie et de la pathologie forestière. Ce laboratoire de diagnostic qui relève du Service des relevés et des diagnostics de la Direction de l'environnement et de la protection des forêts du MRNF offre le service d'identification des insectes et maladies qui s'attaquent aux arbres en forêts naturelles, en plantations et en pépinières forestières. On répond également à des demandes publiques d'identification des ravageurs des arbres. Le laboratoire, la $\mathrm{CIO}$ ainsi que la CCAO peuvent aussi prêter, sur demande, du matériel d'exposition tel que des spécimens d'insectes et de maladies, des cultures de champignons et des montages photos. L'équipe actuelle du laboratoire est composée de Louise Innes, Solange Simard (CCAQ), Lucie Marchand, Lina Breton, Céline Piché (CIQ), Claudyne Dussault, Marc Blouin et Pier-Olivier Carrière-Pagé. Le laboratoire ainsi que les collections traitent en moyenne plus de 6500 échantillons annuellement.

Louise Innes est biologiste et détient une maîtrise (M.Sc.) en biologie végétale de l'Université Laval. Elle travaille au Service des relevés et des diagnostics depuis 27 ans. Elle est membre de la Société de protection des plantes du Québec depuis une vingtaine d'années. Elle a été collaboratrice aux deux dernières éditions du volume Noms des maladies des plantes au Canada.

Laboratoire de diagnostic en phytoprotection du Québec, ministère de I'Agriculture, des Pêcheries et de I'Alimentation du Québec (MAPAQ)

Le Laboratoire de diagnostic en phytoprotection du Québec du ministère de l'Agriculture, des Pêcheries et de I'Alimentation du Québec (MAPAQ), situé au Complexe scientifique, a été créé le 12 mai 1986 en réponse à une épidémie de maladies des racines qui affectait la tomate de serre. Les producteurs de tomates de serre désiraient un diagnostic précis des maladies des racines comme la fusariose (pourriture fusarienne) des racines et du collet et la maladie des racines liégeuses. Aucun service certifié de diagnostic en agriculture n'existait au Québec de sorte que les producteurs devaient expédier leurs échantillons à la Plant Disease Clinic de Guelph, en Ontario, laquelle privilégiait les échantillons des producteurs de cette province. Avant la mise en place du Laboratoire de diagnostic en phytoprotection du Québec et afin d'aider les producteurs québécois, les phytopathologistes du MAPAQ, MM. Alain Devaux, Léon-M. Tartier et Pierre-O. Thibodeau, ainsi que I'entomologiste $\mathrm{Dr}$ Claude Ritchot, réalisaient les diagnostics, et ce, en complémentarité de leur mandat de recherche.

En 1986, le Dr Gilles Émond, alors directeur du Service de phytotechnie de Québec, fut l'initiateur et le fondateur de ce service. II en a assuré la direction jusqu'en 1992. L'équipe de base en phytopathologie était composée de deux phytopathologistes, $M$. Michel Lacroix, président de la Société de protection des plantes du Québec en 1991-1992, et M. Gérard Gilbert, président de la Société pendant deux mandats de 1995 à 1997, et de deux techniciens, M. Mario Tésolin et Mme Lise Vézina. Durant la première saison du laboratoire, M. Pierre-O. Thibodeau accom- 
pagnait régulièrement les phytopathologistes dans la réalisation de diagnostics et par le transfert de son expertise exceptionnelle. Par la suite, Mme Carolle Fortin s'est jointe à l'équipe pour assurer la responsabilité du secrétariat. Actuellement, l'équipe permanente en phytopathologie est constituée de Gérard Gilbert, Dominique Hamel, Cindy Dallaire, Lise Vézina, Mario Tésolin, Chantal Malenfant, Marion Berrouard et Carolle Fortin. Au fil des ans, d'autres personnes ont travaillé et ont contribué au succès de ce service du MAPAQ, soit Michel Lacroix, Lucie Laverdière, Marlène Roger, Ann-Marie Breton, Lise Gauthier, Conrad Beaumont, Diane-J. Cossette, Anne Lahaie et Denise Chénard. Dès les premières années, la détection du Phytophthora fragariae, oomycète responsable de la stèle rouge chez les plants de fraisiers et du pourridié phytophthoréen chez les plants de framboisiers certifiés, alors effectuée par I'équipe de Pierre-O. Thibodeau, a été confiée au Laboratoire de diagnostic en phytoprotection. De plus, dans le cadre de la Loi sur la prévention des maladies de la pomme de terre, le Laboratoire de diagnostic en phytoprotection a été désigné laboratoire officiel pour les analyses et le diagnostic du flétrissement bactérien de la pomme de terre dans les échantillons provenant des territoires protégés. Dès la fondation du laboratoire, des efforts soutenus ont été déployés pour intégrer, adapter et développer diverses techniques de laboratoire sur lesquelles repose toujours l'identification des problèmes phytosanitaires. Cette décision d'appuyer les diagnostics sur les résultats de tests de laboratoire se voulait la signature témoignant de la rigueur et de la précision des identifications réalisées par la section phytopathologie du Laboratoire de diagnostic en phytoprotection du Québec.

Le Dr Claude Ritchot a été le premier entomologiste à réaliser les diagnostics des insectes ravageurs. II était secondé par sa technicienne, Mme Marcelle Parr. Par la suite, des individus ou des duos se sont succédé, soit Josée Boisclair et Claire Fecteau, puis le Dr Jean Denis Brisson et, finalement, la Dre Michèle Roy et Mario Fréchette.

L'identification des plantes nuisibles était confiée à I'équipe de l'Herbier du Québec composée de Claude J. Bouchard, Romain Néron et Louise Guay. Madame Danielle Bernier réalisait et réalise toujours I'identifi- cation des dommages de phytotoxicité des herbicides. En ce qui concerne l'identification des plantes nuisibles, elle est toujours réalisée par Romain Néron.

Initialement, le service était gratuit et réservé exclusivement aux conseillers agricoles du MAPAQ. Une tarification a été instaurée depuis. Environ 700 échantillons ont été traités dès la première année (1986), comparativement à 3500 en moyenne par année actuellement.

Réseau d'avertissements phytosanitaires, ministère de l'Agriculture, des Pêcheries et de I'Alimentation du Québec (MAPAQ)

On doit au Dr Gilles Émond la création du Réseau d'avertissements phytosanitaires (RAP) du MAPAO en 1975. Le Dr Émond en fut le responsable de 1975 à 1992. Le premier coordonnateur fut Léo Raynault, agronome-entomologiste. Lui ont succédé MM. René Couture, Michel Letendre, Serge Bégin, Rémy Fortin, Michel Lacroix et Bruno Gosselin, coordonnateur depuis l'automne 2008. Information tout aussi inédite, les premiers coordonnateurs des premiers réseaux furent Alain Devaux et Jean-Léon Sauvageau: légumes; Michel O'Connell Guibord: légumineuses; Marcel Mailloux: pommier; Gérard Rioux: tabac; Claude Ritchot: maïs; Léon-M. Tartier: pomme de terre; Pierre-O. Thibodeau: cultures en serres; PierreO. Thibodeau: petits fruits; et Michel Letendre: graminées fourragères/grandes cultures. Le RAP est sous la responsabilité de la Direction de l'innovation scientifique et technologique du MAPAQ. En 2008, le RAP est composé de 13 réseaux et secondé par une équipe multidisciplinaire d'avertisseurs et de spécialistes hautement qualifiés. II y a au bas mot plus de 150 collaborateurs répartis sur tout le territoire québécois, force vive essentielle de la protection des cultures contre les maladies biotiques ou abiotiques, les insectes et les plantes nuisibles.

Michel Letendre a complété un baccalauréat (1967) et une maîtrise en sciences biologiques avec spécialisation en entomologie (1970) à I'Université de Montréal. II a d'abord occupé le poste d'entomologiste-chercheur au Service de recherche en défense des cultures du MAPAQ de 1974-1987. Ses travaux ont porté sur la bioécologie et la lutte biologique aux

1. J.-Champlain Perrault (1903-1988). Phytopathologiste, il fut président de la Société de protection des plantes du Québec (1942 à 1944) et de la Société canadienne de phytopathologie. Dès les années 1930, il fut reconnu comme leader de la phytopathologie au Québec et autorité dans le domaine de la pathologie de la pomme de terre, notamment des maladies virales. J.-Champlain Perrault a soutenu sa thèse de doctorat sur le flétrissement bactérien de la pomme de terre en 1946. Membre fondateur de la Corporation des agronomes (1937) et président de l'Ordre des agronomes en 1962, on lui a décerné l'Ordre du Mérite agronomique en 1966 (Généreux et Richard 1989).

2. Elzéar Campagna (1898-1987). Phytopathologiste et spécialiste des plantes nuisibles, il fut président de la Société de protection des plantes du Québec (1938 à 1940). Il a enseigné la botanique, la pathologie maraîchère et la microbiologie dès les années 1920. II a poursuivi des études en pathologie à Cornell University et obtenu un doctorat en sciences naturelles de I'Université Laval en 1940. II fut spécialiste de I'aérobiologie et leader de campagnes d'éradication de I'herbe à poux (Ambrosia spp.). Il fut vice-président fondateur de la Corporation des agronomes (1937), Commandeur de l'Ordre national du Mérite agricole (1985) et doyen de la Faculté d'agriculture de I'Université Laval à La Pocatière. On retrouve des articles et des résumés de communications en mycologie par les phytopathologistes mycologues J.-C. Perrault et E. Campagna dans le tout premier volume des Annales de I'ACFAS, datant de 1937 (Estey 1994).

Estey, R.H. 1994. Essays on the early History of Plant Pathology and Mycology in Canada. McGill-Queen's University Press, Montréal, Canada. $384 \mathrm{p}$.

Généreux, H.J. et C. Richard. 1989. J.-Champlain Perrault, 1903-1988. Can. J. Plant Pathol. 11: 445. 


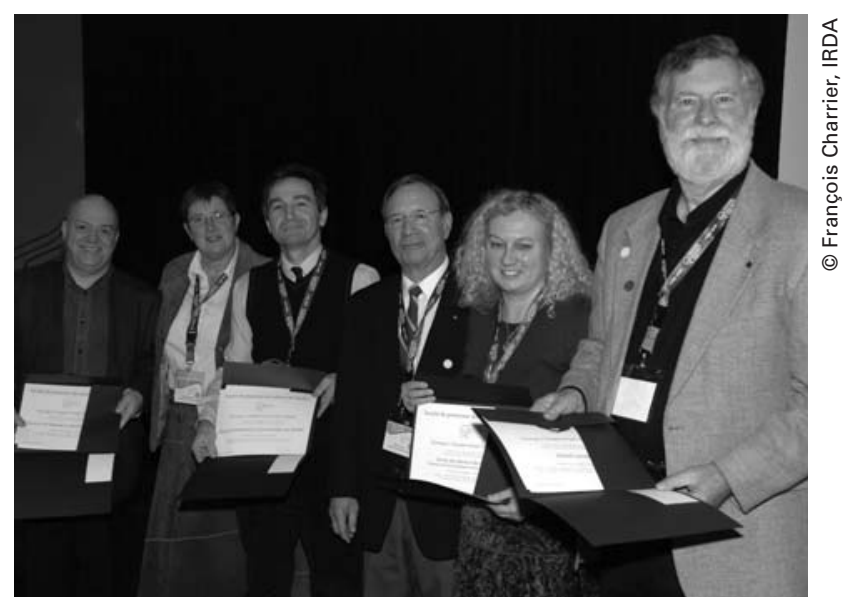

Michel Lacroix, Danielle Bernier, Gérard Gilbert, Gilles Émond, Louise Innes et Michel Letendre

ravageurs. Premier avertisseur responsable du Réseau graminées fourragères/grandes cultures (1975-1990), Michel Letendre est devenu coordonnateur du Réseau d'avertissements phytosanitaires au Service de recherches en phytotechnie de Québec de la Direction des services technologiques du MAPAO en 1987. Il y est demeuré jusqu'en 1998. De 1998 à 2004, Michel a œuvré à titre de spécialiste en gestion intégrée des ennemis des cultures et répondant en matière de pesticides pour le MAPAQ, à la Direction des services technologiques. II a coordonné le Programme d'homologation de pesticides pour usages mineurs et le Programme d'homologation d'urgence, à l'échelle provinciale (Québec). II a très activement fait la promotion et supporté le développement de la lutte intégrée au Québec. Ainsi, il a siégé au Comité de concertation et de suivi de la Stratégie phytosanitaire du Québec. Michel Letendre a occupé la présidence du Comité canadien d'experts en lutte intégrée (CELI) de 1999 à 2003. II a été successivement président de la Société de protection des plantes du Québec en 1988-1989 et président de la Société d'entomologie du Québec en 1995-1996. Ses qualités remarquables ont été reconnues et soulignées. Michel est membre du Club des Ambassadeurs du Palais des Congrès de Montréal pour I'organisation du Congrès conjoint Entomological Society of America, Société d'entomologie du Canada et Société d'entomologie du Québec. II est récipiendaire du Méritas horticole 2004 du Conseil québécois de I'horticulture. Depuis sa retraite en 2004, la phytoprotection occupe toujours une part importante de ses activités, en particulier par sa participation au programme fédéral de réduction des risques liés aux pesticides. II agit aussi à titre de consultant en matière de protection des cultures pour I'Association des jardiniers-maraîchers du Québec (AJMQ).

À l'occasion de la commémoration de son centenaire (1908-2008), la Société de protection des plantes du Québec est fière de présenter I'hommage J.Champlain-Perrault ${ }^{1} /$ Elzéar-Campagna $^{2}$ - Services publics en phytoprotection au Service des relevés et des diagnostics, au Laboratoire de diagnostic en phytoprotection du Québec et au Réseau d'avertissements phytosanitaires, en gage de sa plus haute gratitude pour leur contribution inestimable à la protection des cultures et des forêts du Québec.

Hommage remis par le Dr Gilles Émond, président du comité du centenaire et lauréat d'un prix Excellence pour l'ensemble des réalisations. Dans le cadre de ses fonctions de gestionnaire, le Dr Émond a été le maître d'œuvre de la mise sur pied du Réseau d'avertissements phytosanitaires ainsi que du Laboratoire de diagnostic en phytoprotection du Québec.

\section{Hommage Victor-A.-Huard/Georges-Maheux Pionnier et Force vive de l'entomologie forestière Jacques Régnière}

Jacques Régnière est chercheur scientifique au Centre de foresterie des Laurentides (CFL) du Service canadien des forêts (SCF), à Québec. Diplômé de I'Université Laval en biologie (1976), Jacques Régnière détient un doctorat en entomologie avec spécialisation en écologie et biomathématique de North Carolina State University (1980). Chercheur scientifique sénior au SCF depuis 1980, il est aussi professeur associé au Département des sciences du bois et de la forêt de I'Université Laval et à la Faculté de foresterie de I'Université de Toronto, où il dirige des étudiants des cycles supérieurs. Spécialiste de l'écologie quantitative, ses recherches portent sur la dynamique des populations d'insectes forestiers, dont la tordeuse des bourgeons de l'épinette, le dendroctone du pin et la spongieuse, sur la gestion inté- grée, la saisonnalité et l'impact des changements climatiques sur ces organismes. II est l'auteur de très nombreux articles scientifiques.

Le Dr Régnière a mis au point le logiciel BioSIM pouvant prédire le développement saisonnier des insectes. Ce système est utilisé dans plusieurs pays comme outil d'optimisation des programmes de lutte contre les ravageurs et aussi dans la prédiction des impacts des changements climatiques sur la distribution géographique et les dommages causés par les insectes nuisibles. Les travaux du Dr Régnière sur la dynamique des populations de la tordeuse des bourgeons de l'épinette, mettant en lumière les mécanismes rétroactifs reliant la performance de l'insecte à la structure des peuplements, ont conduit à une révision en profondeur des concepts de base expli- 
quant le comportement démographique de cet insecte et l'élaboration de méthodes d'intervention préventive et hâtive pour en minimiser les dommages. Le Dr Régnière a mis au point des modèles permettant d'optimiser l'efficacité des programmes de lutte contre la tordeuse et la spongieuse à l'aide de I'insecticide biologique Bacillus thuringiensis. Les nombreux outils mis au point par le Dr Régnière servent à un grand nombre d'organisations au Canada, aux États-Unis, en Amérique du Sud, en Europe, en Asie et en Nouvelle-Zélande. Le Dr Régnière est reconnu internationalement comme expert en modélisation de la dynamique des populations d'insectes et en évaluation des risques posés par les espèces envahissantes et les changements climatiques. II a été invité à prononcer plus de 30 conférences à des événements internationaux tenus à l'étranger. II a siégé à un panel d'experts de la FAO sur les changements climatiques et les espèces envahissantes transfrontalières. On lui a accordé trois fois la Prime au mérite du SCF pour la créativité, I'innovation et le travail d'équipe.
À l'occasion de la commémoration de son centenaire (1908-2008), la Société de protection des plantes du Québec est heureuse de présenter I'hommage Victor-A.-Huard $1 /$ Georges-Maheux ${ }^{2}-$ Pionnier et Force vive de I'entomologie forestière au $\mathrm{Dr}$ Jacques Régnière en gage de gratitude pour sa contribution inestimable au rayonnement de l'entomologie forestière et à la protection des forêts.

L'hommage Victor-A.-Huard/Georges-Maheux a été décerné au Dr Jacques Régnière le 21 novembre 2008 par M. Guy Bélair, président de la SPPQ, lors du Symposium "La phytoprotection, 100 ans de découvertes: un tremplin vers l'avenir! ", dans le cadre de la réunion annuelle conjointe SPPQ-SEQ, tenue à I'Hôtel Château Laurier. L'entomologie forestière est un champ d'activité et de recherche de la phytoprotection qui revêt un caractère fondateur (Victor-A. Huard) et pionnier (Georges Maheux) pour la Société de protection des plantes du Québec. L'attribution de cet hommage en entomologie forestière scelle les liens qui, en ce $100^{\circ}$ anniversaire de la SPPQ, unissent la Société de protection des plantes du Québec et la Société d'entomologie du Québec.

1. Victor-Alphonse Huard (1853-1929). Entomologiste, il fut membre fondateur et membre honoraire (1916) de la SPPQ. Premier entomologiste attitré de la province de Québec, de 1913 à 1916, et "disciple" de Léon Provancher, Victor-A. Huard a repris en 1894 la publication du Naturaliste canadien qui avait cessé de paraître en juin 1891. La collection entomologique de Victor-A Huard a été restaurée et elle a été déposée aux Collections de l'Université Laval en 2003 (Perron 2005).

2. Georges Maheux (1889-1977). Entomologiste, il fut président (1930 à 1932) et assistant secrétaire-trésorier (1932 à 1939) de la SPPQ. Pionnier de l'entomologie, c'est l'une des plus grandes figures que l'entomologie québécoise ait connue. II a succédé à Victor-A. Huard comme entomologiste attitré de la province de Québec à compter de 1916. Ingénieur forestier, Georges Maheux enseigna la phytopathologie et l'entomologie forestière à I'Université Laval. II fut président de I'ACFAS en 1935; membre fondateur et secrétaire-trésorier de la Corporation des ingénieurs forestiers et président de la section de Québec de la Corporation des agronomes; président de la Société d'entomologie de I'Ontario; et président de la Société d'entomologie du Canada. La Société d'entomologie du Québec (SEQ) a donné son nom au concours de rédaction scientifique destiné à ses membres étudiants. L'Université de Montréal lui a décerné un doctorat honoris causa en 1937 et l'Université Laval a fait de même en 1942. II fut Commandeur du Mérite agricole (1941), membre de la Société royale du Canada (1944), ainsi que membre honoraire (1953) et premier membre émérite (1973) de la SEQ (Perron 2006).

Perron, J.-M. 2005. La collection d'insectes de Victor Alphonse Huard. Nat. Can. 129: 30-34.

Perron, J.-M. 2006. Georges Maheux (1889-1977), une longue et brillante carrière consacrée à l'entomologie. Antennae 13 (2): $14-18$ 